\title{
RESEARCH
}

Open Access

\section{The microbial metabolite $p$-Cresol induces autistic-like behaviors in mice by remodeling the gut microbiota}

Patricia Bermudez-Martin ${ }^{1}$, Jérôme A. J. Becker ${ }^{2,3}$, Nicolas Caramello ${ }^{1,4}$, Sebastian P. Fernandez ${ }^{1}$, Renan Costa-Campos ${ }^{1}$, Juliette Canaguier', Susana Barbosa', Laura Martinez-Gili ${ }^{5}$, Antonis Myridakis ${ }^{5}$, Marc-Emmanuel Dumas ${ }^{5,6,7,8}$, Aurélia Bruneau ${ }^{9}$, Claire Cherbuy ${ }^{9}$, Philippe Langella9 ${ }^{9}$ Jacques Callebert ${ }^{10,11}$, Jean-Marie Launay ${ }^{10,11}$, Joëlle Chabry', Jacques Barik, Julie Le Merrer ${ }^{2,3}$, Nicolas Glaichenhaus ${ }^{1,12}$ and Laetitia Davidovic ${ }^{1,12^{*}}$

\begin{abstract}
Background: Autism spectrum disorders (ASD) are associated with dysregulation of the microbiota-gut-brain axis, changes in microbiota composition as well as in the fecal, serum, and urine levels of microbial metabolites. Yet a causal relationship between dysregulation of the microbiota-gut-brain axis and ASD remains to be demonstrated. Here, we hypothesized that the microbial metabolite $p$-Cresol, which is more abundant in ASD patients compared to neurotypical individuals, could induce ASD-like behavior in mice.
\end{abstract}

Results: Mice exposed to $p$-Cresol for 4 weeks in drinking water presented social behavior deficits, stereotypies, and perseverative behaviors, but no changes in anxiety, locomotion, or cognition. Abnormal social behavior induced by $p$-Cresol was associated with decreased activity of central dopamine neurons involved in the social reward circuit. Further, $p$-Cresol induced changes in microbiota composition and social behavior deficits could be transferred from $p$-Cresol-treated mice to control mice by fecal microbiota transplantation (FMT). We also showed that mice transplanted with the microbiota of $p$-Cresol-treated mice exhibited increased fecal $p$-Cresol excretion, compared to mice transplanted with the microbiota of control mice. In addition, we identified possible $p$-Cresol bacterial producers. Lastly, the microbiota of control mice rescued social interactions, dopamine neurons excitability, and fecal $p$-Cresol levels when transplanted to $p$-Cresol-treated mice.

Conclusions: The microbial metabolite $p$-Cresol induces selectively ASD core behavioral symptoms in mice. Social behavior deficits induced by $p$-Cresol are dependant on changes in microbiota composition. Our study paves the way for therapeutic interventions targeting the microbiota and $p$-Cresol production to treat patients with ASD.

Keywords: Microbiota, Autism, Behavior, Reward system, Metabolite, $p$-Cresol, 4-Cresol

\footnotetext{
* Correspondence: davidovic@ipmc.cnrs.fr

${ }^{1}$ Institut de Pharmacologie Moléculaire et Cellulaire, Centre National de la

Recherche Scientifique, Université Côte d'Azur, 660 route des Lucioles, 06560

Valbonne, France

${ }^{12}$ Fondation FondaMental, Créteil, France

Full list of author information is available at the end of the article
}

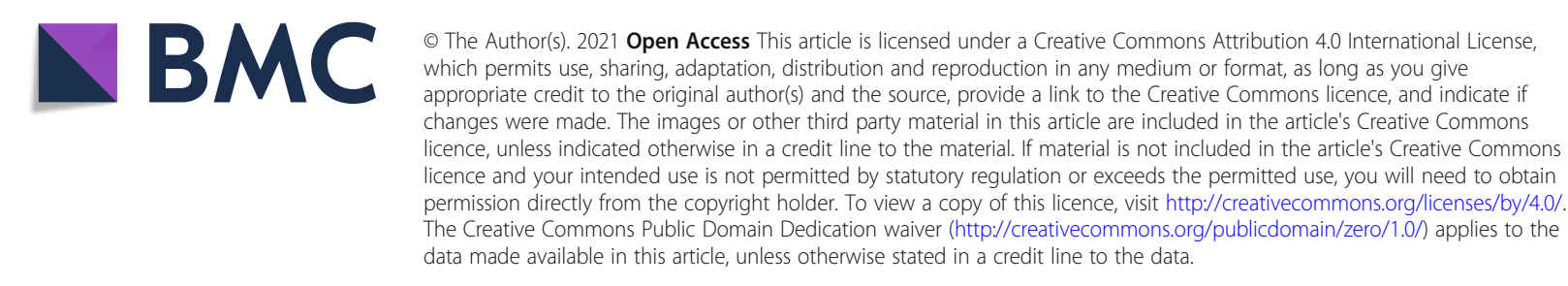




\section{Introduction}

ASD are frequent neurodevelopmental disorders resulting from interactions between genetic predisposition and environmental risks [1]. ASD median prevalence worldwide is estimated to be 1\% [2]. While $10-25 \%$ of ASD cases are explained by mutations in specific genetic loci, twin studies have revealed that genetic and environmental factors share equal influence on ASD risk [3]. The identification of environmental factors contributing to ASD is therefore critical to better understand their etiology. ASD core symptoms encompass social interaction and communication deficits, perseverative/stereotyped behaviors, restricted interests, and abnormal sensory processing [4]. Also, ASD often co-occur with anxiety disorder, hyperactivity and intellectual disability (ID) [4]. A growing body of evidence supports an involvement of the gut microbiota-brain axis in ASD [1]. First, ASD is associated with gastrointestinal (GI) dysfunction and increased intestinal permeability [1]. Second, children with ASD and concurrent GI symptoms exhibit more pronounced social impairments, sensory over-responsivity, and anxiety compared to ASD peers without GI symptoms [5-7]. Third, ASD patients exhibit gut microbiota dysbiosis. Indeed, bacterial $\beta$-diversity changes associated with increased and decreased abundances of the genera Clostridioides and Bifidobacterium respectively have been reported in three independent meta-analyses [8-10]. In a pilot study, fecal microbiota transplantation (FMT) from healthy individuals to ASD patients durably alleviated both GI symptoms and ASD core symptoms [11]. Fourth, dysbiosis in ASD patients is associated with altered levels of urinary, plasmatic, or fecal microbial metabolites such as methylamines, indoles, and tyrosinederived metabolites [12-19].

Altered microbiota composition [20-24] and abnormal levels of microbial metabolites [20, 23, 24] have also been observed in rodent models of ASD: the maternal immune activation (MIA), the diet-induced obesity (DIO) and the valproate environmental models, the BTBR idiopathic model, and the Shank3b-KO genetic model. Further, changes in microbiota composition induced by FMT or probiotic treatment alleviated behavioral alterations in several of these ASD models [20-22]. Finally, mice born from mothers transplanted with feces from ASD patients exhibited social behavior deficits, changes in microbiota composition, and abnormal patterns of microbial metabolites [25]. Several microbial metabolites were shown to induce behavioral changes when administered to rodents. Treatment with the short-chain fatty acid (SCFA) propionate induced social interaction deficits, stereotypies, cognitive deficits, and anxiety in rats [17]. The tyrosine degradation product 4ethylphenylsulfate (4-EPS) and indole induced anxiety in mice $[1,20,26]$. Altogether, these data suggested that dysbiosis could contribute to ASD core and associated symptoms via the production of microbial metabolites.

Among the microbial metabolites linked to ASD, the small aromatic metabolite $p$-Cresol (para-Cresol, 4Cresol, 4-methylphenol) was consistently found at increased levels in the urine and feces of ASD patients [14, $15,18,19,27,28]$. Also, $p$-Cresol urinary levels correlated with the severity of ASD behavioral alterations [14, 15]. No reports of elevated $p$-Cresol in the blood of ASD patients are available, either because it was not detected or not assessed in clinical studies on plasma or serum of ASD patients. Although environmental exposure to $p$ Cresol is relatively common and occurs through the skin, as well as the GI and respiratory systems [29], the largest and most widespread source of this compound results from tyrosine degradation by the intestinal microbiota in the colon. At least 55 bacterial strains present in the human microbiota can produce $p$-Cresol. These strains are phylogenetically divergent and spread across the Bifidobacteriaceae, Enterobacteriaceae, Coriobacteriaceae, Bacteroidaceae, Fusobacteriaceae, Lactobacillaceae, and Clostridiaceae families [30]. It was also shown that $p$-Cresol conferred a selective growth advantage to Clostridioides difficile [31], possibly contributing to the overabundance of members of the Clostridioides genus in ASD patients [8-10]. However, to date, the causal relationship between microbiota dysbiosis, $p$-Cresol production, and ASD symptoms has not been demonstrated. Here, we show that mice chronically exposed to $p$-Cresol presented social behavior deficits, stereotyped/perseverative behaviors, changes in microbiota composition, and elevated $p$-Cresol levels. Further FMT experiments revealed that $p$-Cresol induced social deficits by a microbiota-dependent mechanism.

\section{Results}

Mice exposed to $p$-Cresol exhibit ASD-like behaviors that persist after treatment discontinuation

To mimic exposure to $p$-Cresol through the GI tract, we treated C57BL/6J male mice with $p$-Cresol in drinking water starting at 4.5 weeks of age (Fig. 1A, Additional file 1: Fig. S1A). A treatment for 4 weeks with $p$-Cresol did not induce changes in body weight, drink or food intake (Additional file 1: Fig. S1B-D). Compared to untreated control animals, $p$-Cresol-treated mice exhibited increased levels of $p$-Cresol in urine and feces (Additional file 1: Fig. S1E, F), but not in serum (Additional file 1: Fig. S1G).

$p$-Cresol-treated mice were subjected to behavioral phenotyping (Fig. 1A) for social interactions and repetitive/perseverative behaviors (as proxies for ASD core symptoms) (Fig. 1B-N, Additional file 1: Fig. S2A-L) as well as anxiety, hyperactivity, and cognitive deficits (as proxies of ASD comorbidities) (Additional file 1: Fig. 


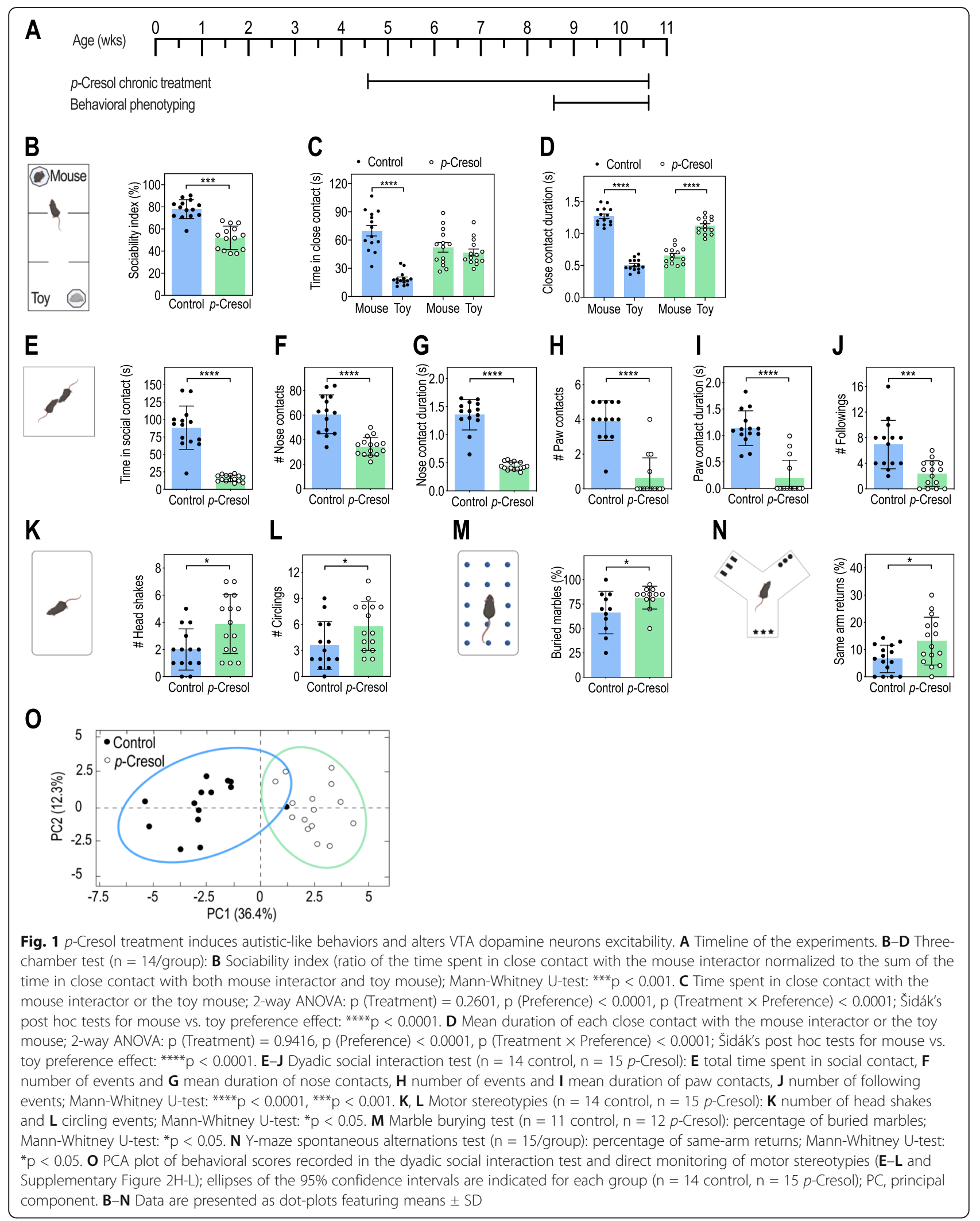


S2M-U). In the 3-chamber test (Additional file 1: Fig. S2A-G, Fig. 1B-D), $p$-Cresol-treated mice presented reduced sociability (Fig. 1B) and no preference for the mouse interactor towards the toy mouse (Fig. 1C) compared to control mice. Although the number of close contacts with the mouse interactor was higher than with the toy mouse (Additional file 1: Fig. S2G), their mean duration was reduced (Fig. 1D). During dyadic social interactions, $p$-Cresol-treated mice spent less time in social contact compared to control mice (Fig. 1E). The time, number, and mean duration of both nose and paw contacts as well as the number of followings were also reduced (Fig. 1F-J, Additional file 1: Fig. S2H, I) demonstrating that $p$-Cresol exposure impaired social interactions.

As for repetitive/perseverative behaviors, while $p$-Cresol-treated mice exhibited similar numbers of rearing episodes and time spent in digging and self-grooming compared to control mice (Additional file 1: Fig. S2J-L), they displayed more frequent head shakes and circling events (Fig. 1K, L). An increase in stereotyped behaviors in $p$-Cresol-treated mice was confirmed in the marble burying test (Fig. 1M). p-Cresol-treated mice also exhibited increased frequency of perseverative same-arm returns in the Y-maze spontaneous alternation task (Fig. 1N). Finally, $p$-Cresol-treated mice were clearly separated from control mice along the first principal component (PC1) axis in a PCA analysis of scores recorded in dyadic social interaction and direct monitoring of stereotypies (Fig. 1O).

As for other behaviors, $p$-Cresol-treated and control mice displayed similar nocturnal and diurnal locomotor activity as assessed in actimetry chambers, and traveled the same distance in the open-field (Additional file 1: Fig. S2M-O), showing that $p$-Cresol exposure did not induce hyperactivity. Also, the number of entries and time spent in the open-field center, the latency to feed in the novelty-suppressed feeding test, and the percentage of time spent in the open arm of the zero-maze were not impacted (Additional file 1: Fig. S2P-S), suggesting that $p$-Cresol exposure did not induce anxiety. Finally, $p$-Cresol-treated mice explored objects similarly to control mice and displayed a similar recognition index for the novel object in the novel object recognition task (Additional file 1: Fig. S2T, U), indicating that their cognitive ability were preserved.

We then investigated whether $p$-Cresol-induced social deficits and stereotypies were abolished when the treatment was discontinued (Additional file 1: Fig. S3A). After a 4-week washout, the effects of $p$-Cresol on social interactions and stereotyped/perseverative behaviors persisted and were of similar magnitude as shown Fig. S3BO (Additional file 1). PCA analysis of social behavior and stereotypies scores revealed that control and $p$ -
Cresol-treated mice were clearly separated along the PC1 axis, both pre- and post-washout (Additional file 1: Fig. S3P). Altogether, these results suggested that $p$-Cresol selectively induced ASD core symptoms which persisted durably after $p$-Cresol exposure.

\section{p-Cresol impairs dopamine neurons excitability in the ventral tegmental area (VTA)}

We then investigated the impact of $p$-Cresol exposure on electrophysiological properties of dopamine neurons in the lateral VTA, known to project to the nucleus accumbens medial shell as part of a "socially engaged reward circuit" [32]. Altered VTA connectivity and impaired VTA dopamine neurons excitability were observed in both ASD patients [33] and mouse models of ASD [21, 22, 34-37]. We used whole-cell patch-clamp to record VTA dopamine neurons in acute brain slices from control and $p$-Cresol-treated mice (Fig. 2A, B). First, $p$-Cresol-treated mice displayed reduced excitability of VTA dopamine neurons, with a reduction in the number of evoked action potentials (Fig. 2C-E). Second, both the amplitude and frequencies of miniature spontaneous excitatory post-synaptic currents (sEPSC) were reduced in VTA dopamine neurons of $p$-Cresol-treated animals compared to controls (Fig. 2F-J). Therefore, $p$ Cresol treatment resulted in decreased activity of VTA dopamine neurons.

\section{$p$-Cresol impacts the gut microbial ecology}

Because $p$-Cresol exposure affects bacterial diversity [31], we analyzed the bacterial composition of the fecal microbiota in $p$-Cresol-treated and control mice using $16 \mathrm{~S}$ ribosomal RNA (rRNA) sequencing. There was no difference in bacterial richness or evenness between the two groups, as assessed by the observed number of amplicon sequence variant (ASV), Shannon's and Pielou's indexes (Fig. 3A-C). In contrast, $\beta$-diversity analysis based on Aitchison's distances revealed significant divergences in microbial composition between $p$-Cresoltreated and control mice (Fig. 3D). We then sought to identify discriminant features (ASV and bacterial taxa up to the phylum level) using the analysis of composition of microbiomes (ANCOM) method [38] that considers the compositional nature of $16 \mathrm{~S}$ rRNA gene sequencing datasets and controls false discovery rate [39]. We found that $19 \mathrm{ASV}, 4$ species and 2 genera were discriminant (|centered-log ratio $(C L R) \mid>0.2 ; \mathrm{W}=0.7)$ with the largest effect size $(|C L R|>0.5)$ being observed for ASV related to the Bacteroidales, Clostridiales, and Burkholderiales orders (Fig. 3E, Additional file 2: Tab. $\mathrm{S} 1)$. We identified 15 taxonomic features increased in $p$ Cresol-treated mice (10 ASV, 3 species, 2 genera). In particular, $p$-Cresol increased the counts of ASV affiliated to Duncaniella dubosii, one Barnesiella sp., one 


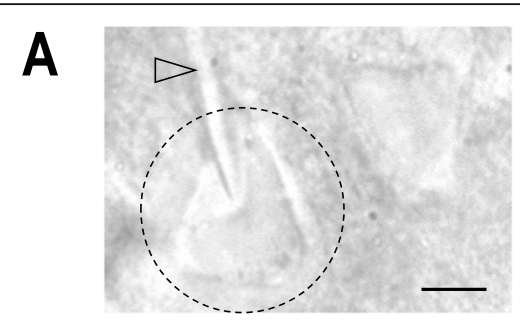

C

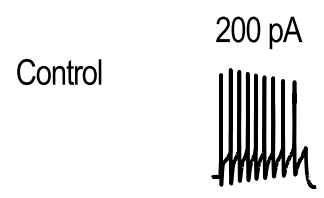

p-Cresol

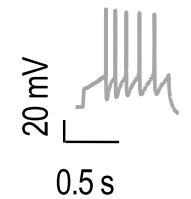

$\mathbf{F}$

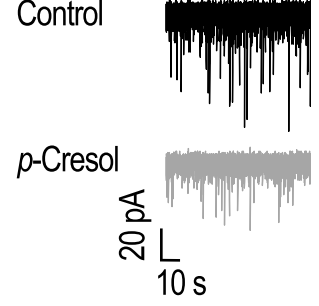

D

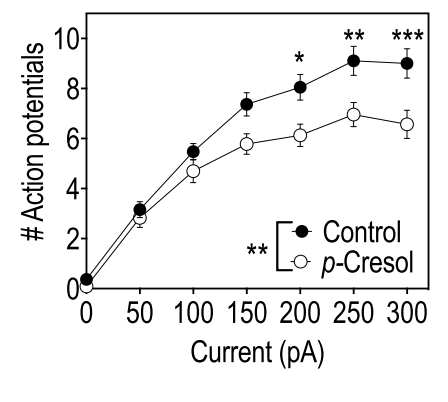

G
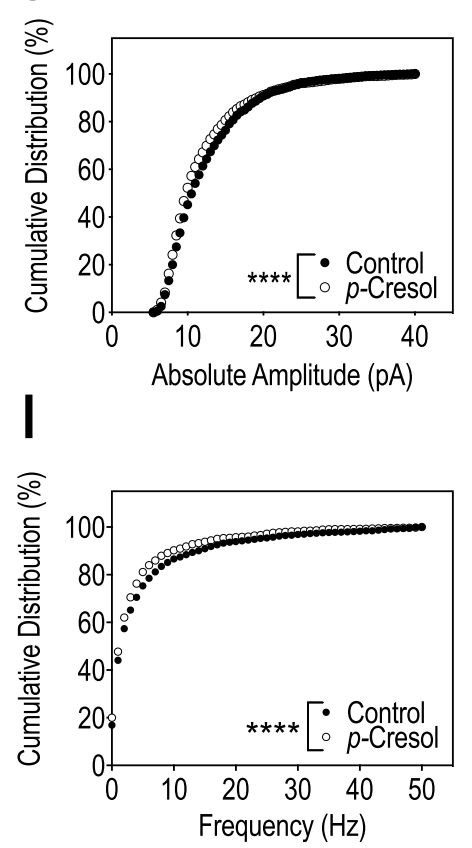

E

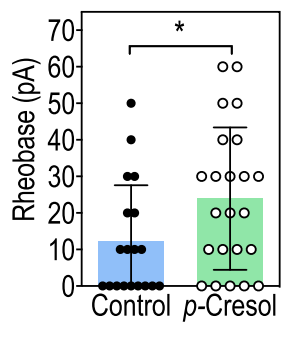

$\mathrm{H}$

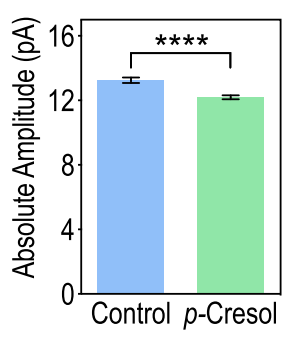

J

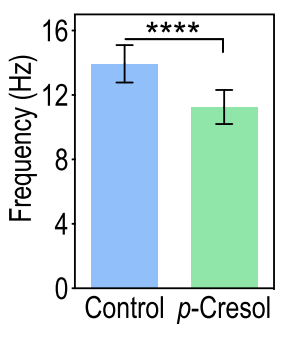

Fig. 2 (See legend on next page.) 


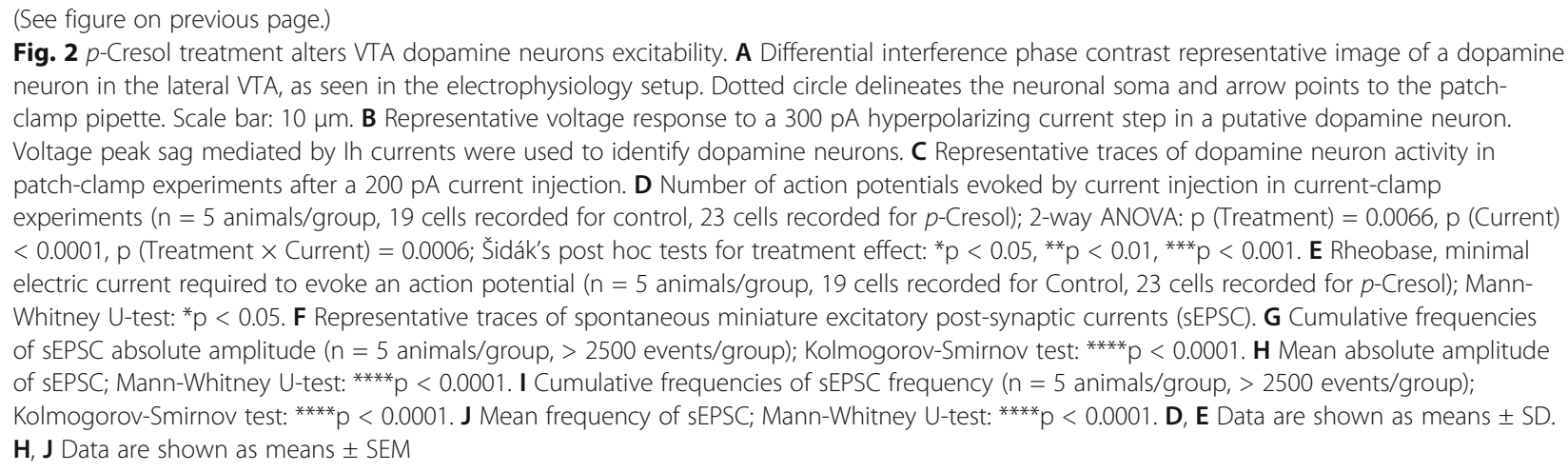

Muribaculaceae bacterium, one Anaerobium sp., and Turicimonas muris (Fig. 3E, F, Additional file 2: Tab. S1). We also identified 10 taxonomic features (9 ASV, 1 species), all affiliated to the Clostridiales order, that were depleted in $p$-Cresol-treated mice (Fig. 3E, Additional file 2: Tab. S1). In particular, decreased counts of ASV affiliated to one Eisenbergiella sp., Lacrimispora saccharolytica, one Clostridiaceae bacterium, Ruthenibacterium lactatiformans, and one Anaerobium sp. were observed in $p$-Cresol-treated mice (Fig. 3E, G, Additional file 2: Tab. S1). Thus, $p$-Cresol-induced changes in microbial composition were mainly observed at the ASV level and scarcely at the species or genus level suggesting that $p$ Cresol induced selective and not broad taxonomic changes in microbial composition. To model how microbiota composition (assessed by ASV pseudocounts) predicted social abilities (Fig. 1E) and stereotypies (Fig. 1K, $\mathrm{L})$, we then used a random forest (RF) regressor blind to the experimental groups. We found that 15 and 13 ASV were the strongest drivers of social abilities (Fig. $3 \mathrm{H}$ ) and stereotypies (Fig. 3I) respectively. Of note, selected ASV explained social behavior scores, with up to $12 \%$ of the score explained by one ASV affiliated to Duncaniella dubosii (Fig. $3 \mathrm{H}$ ), while they contributed less to explain stereotypies with one ASV affiliated to a Clostridiaceae bacterium explaining $5 \%$ of the score (Fig. 3I). These data reinforce the link between microbial composition and ASD-like core symptoms and in particular social behavior.

\section{The microbiota from $p$-Cresol-treated mice induces social behavior deficits when transplanted to untreated recipients}

To investigate whether microbiota composition changes may account for $p$-Cresol-induced behavioral alterations, we transplanted fecal microbiota from either $p$-Cresoltreated or control mice to untreated recipients and assessed their behavior 3 weeks post-FMT (Fig. 4A). For the sake of clarity, we will use FMT ${ }^{\text {Control }}$ as an abbreviation for "transplantation with the microbiota of control mice". Likewise, we will use $\mathrm{FMT}^{p-C r e s o l}$ as an abbreviation for "transplantation with the microbiota of $p$-Cresol-treated mice".

In the 3-chamber test (Additional file 1: Fig. S4A-G, Fig. 4B-D), $\mathrm{FMT}^{p \text {-Cresol }}$ mice presented reduced sociability (Fig. 4B) and no preference for the mouse interactor towards the toy mouse (Fig. $4 \mathrm{C}$ ) compared to FMT ${ }^{\text {Control }}$ mice. Although the number of close contacts with the mouse interactor was higher than with the toy mouse (Additional file 1: Fig. S4G), their mean duration was reduced (Fig. 4D).

In the dyadic social interaction test, compared to $\mathrm{FMT}^{\text {Control }}$ mice, $\mathrm{FMT}^{p-\mathrm{Cresol}}$ mice spent less time in social contact, exhibited decreased time, number, and mean duration of nose and paw contacts, but no change in the number of followings (Fig. 4E-J, Additional file 1: Fig. S4H, I). Social deficits were accompanied by increased number of head shakes (Fig. 4K), but no changes in number of circling episodes (Fig. 4L), and of time spent grooming and digging (Additional file 1: Fig. S4J, $\mathrm{K}) \cdot \mathrm{FMT}^{\text {Control }}$ and $\mathrm{FMT}^{p \text {-Cresol }}$ groups were separated along the PC1 axis in PCA analysis of social interaction and stereotypies scores (Fig. 4M). Further, mice from these two groups behaved similarly in the noveltysuppressed feeding and zero-maze tests (Additional file 1: Fig. S4L, M) suggesting that their anxiety levels were not impacted. To summarize, the transplantation of microbiota from $p$-Cresol-treated mice to untreated recipients recapitulated the effects of $p$-Cresol treatment on social behavior deficits and partially on stereotypies.

\section{Transplantation of the microbiota from $p$-Cresol-treated mice to untreated recipients impacts gut microbial ecology and increased fecal excretion of $p$-Cresol}

We used 16S rRNA sequencing to analyze the microbiota of $\mathrm{FMT}^{\text {Control }}$ and $\mathrm{FMT}^{p-\text { Cresol }}$ mice 3 weeks postFMT. Microbial richness and evenness were similar between the two groups as assessed by $\alpha$-diversity indexes (Fig. 5A-C). However, analysis of $\beta$-diversity based on Aitchison's distances revealed significant divergences in 

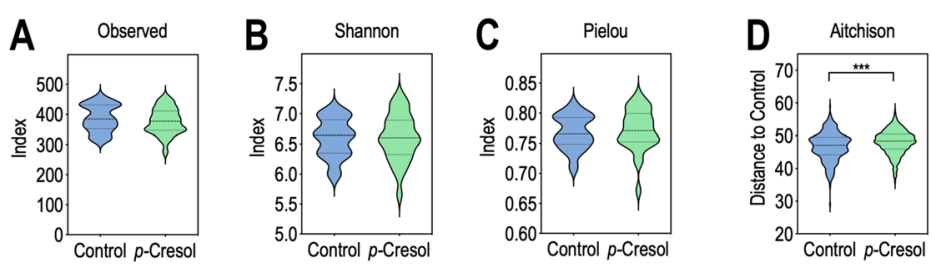

E

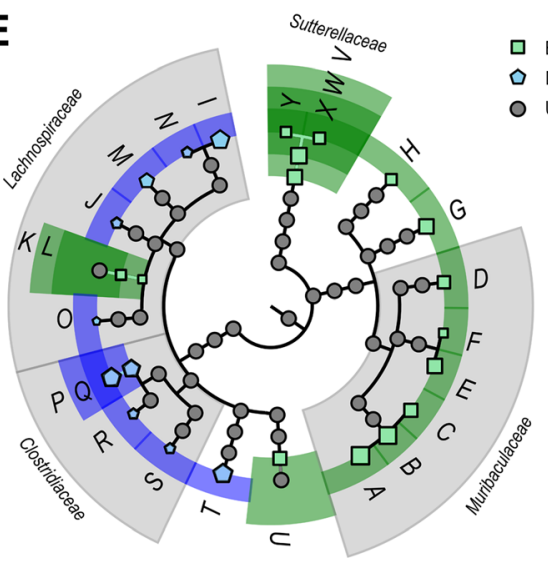

ㅁ Enriched in $p$-Cresol

Depleted in p-Cresol

Unchanged

\section{A: a085_Muribaculum_sp} B: b173_Muribaculum_sp D: 6019 _Duncaniella_dubosii E: e6bd_Muribaculaceae_bacterium F: dfd8_Muribaculaceae_bacterium G: cb42_Culturomica_sp

H: 4b9b_Bamesiella_sp 1: Gaa4_Lacrimispora_saccharolytica $\mathrm{J}$ : ge6d_Eisenbergiella_sp

K: Anaerobium

M: 3041 [Clostridium]_asparagiforme N: bf38_Lacrimispora_saccharolytica O: 488d_Anaerotignum_sp P: Clostridioides sp_Clone-49 R: 785 e Clostridiaceae bacterium S: a199_Clostridiaceae_bacterium T: ae93_Ruthenibacterium_lactatiformans U: Aminipila butyrica

V: Turicimonas

W: Turicimonas_muris

$X$ : ced7_Turicimonas_muris Y: d737_Turicimonas_muris
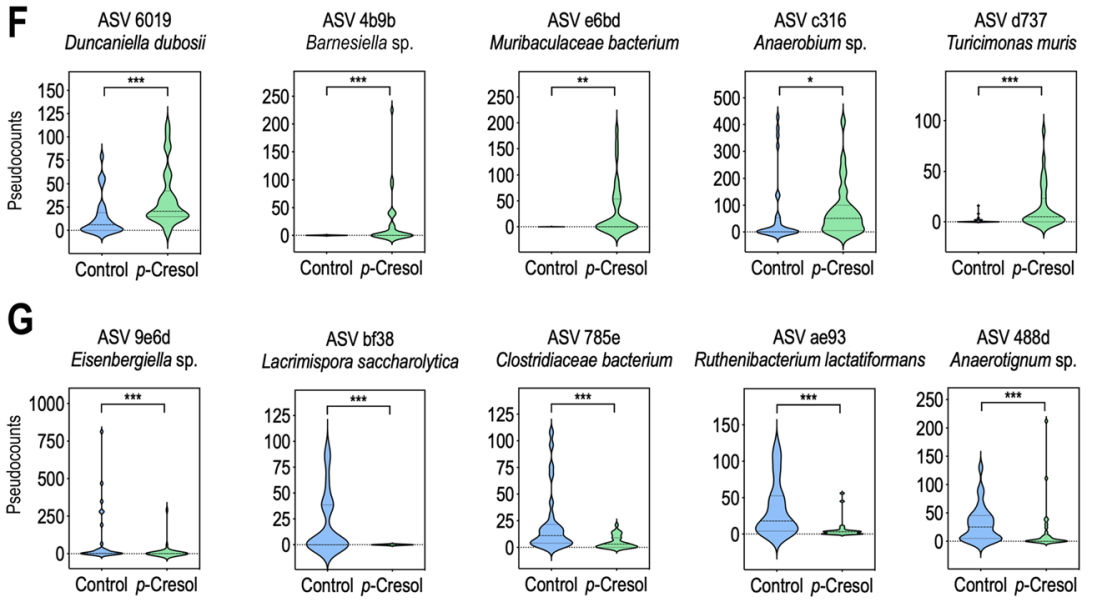

H
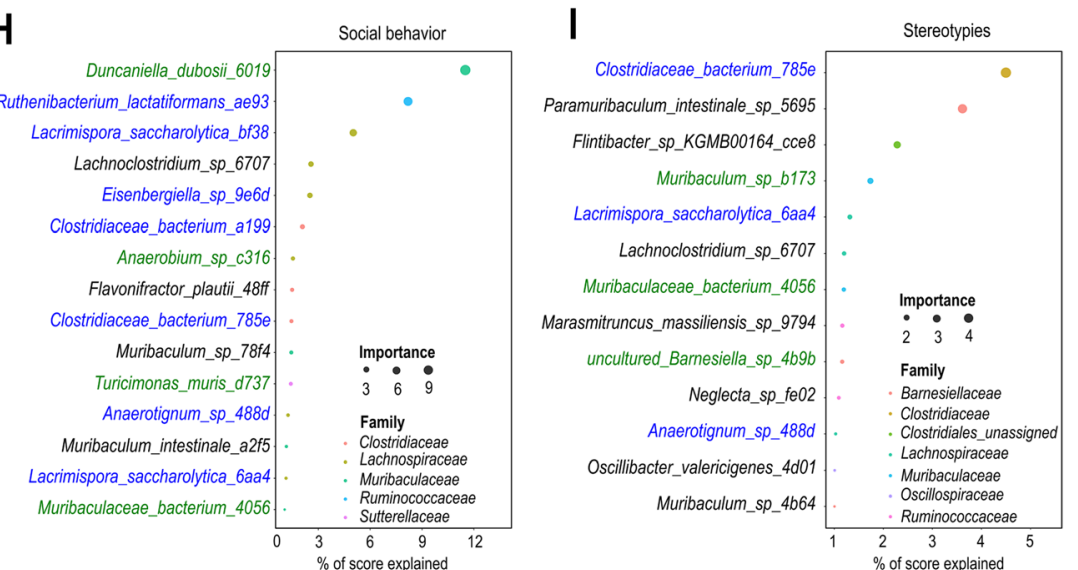

Fig. 3 (See legend on next page.) 
(See figure on previous page.)

Fig. 3 -Cresol impacts microbial composition and selective bacterial taxa predict social deficits and stereotypies. A-C a-diversity as measured by Observed number of ASV (A), Shannon's (B), and Pielou evenness' (C) indexes ( $n=30$ control, $n=29 p$-Cresol); Kruskall-Wallis pairwise test: $p>0.05$. D $\beta$-diversity as measured by Aitchison distances from control and $p$-Cresol groups based on 165 rRNA gene sequencing ( $n=30$ control, $n=29 p$-Cresol). Group differences were tested by pairwise PERMANOVA, $p=0.001$. E Synthetic cladogram presenting dysregulated ASV and bacterial taxa increased (green) or depleted (blue) upon $p$-Cresol exposure identified by ANCOM. Symbol size reflects the value of the corresponding CLR. F, G Pseudocounts plots from selected ASV increased (F) or depleted (G) upon $p$-Cresol exposure identified by ANCOM ( $n=30$ control, $n=29 p$-Cresol); Mann-Whitney U-Test: ${ }^{*} p<0.05$, ${ }^{* *} p<0.01$, ${ }^{* * *} p<0.001$. $\mathbf{H}$, I Microbial composition prediction of behavioral scores. ASV best predicting social behavior (H) or stereotypies (I) as identified by random forest analysis. Only ASV contributing $>1 \%$ accuracy in behavioral scores prediction are presented. ASV identical to or ASV pointing towards the same species as ASV identified by ANCOM as increased or depleted upon $p$-Cresol exposure are labeled in green and blue respectively. A-D, F, G Data are presented as violin plots featuring frequency distribution of data, median (dashed line) and quartiles (dotted line).

microbial composition between FMT $^{\text {Control }}$ and $\mathrm{FMT}^{p \text {-Cresol }}$ mice (Fig. 5D), similarly to what was observed between $p$-Cresol-treated and control mice (Fig. 3D). ANCOM analysis revealed that 22 ASV, 5 species, 3 genera and 1 family were discriminant (|CLR $\mid>$ 0.2; $\mathrm{W}=0.7$ ) (Fig. 5E, Additional file 3: Tab. S2). ASV counts related to Duncaniella dubosii, one uncultured Barnesiella sp., and one Anaerobium sp. were increased in $\mathrm{FMT}^{p \text {-Cresol }}$ mice (Fig. 5E, F, Additional file 3: Tab. S2), as already shown for $p$-Cresol-treated mice (Fig. 3E, F, Additional file 2: Tab. S1). In addition, ASV counts related to one Eisenbergiella sp., Lacrimispora saccharolytica, and one Clostridiaceae bacterium were decreased in $\mathrm{FMT}^{p \text {-Cresol }}$ mice (Fig. 5E, G, Additional file 3: Tab. $\mathrm{S} 2$ ), as already shown for $p$-Cresol-treated mice (Fig. 3E, G, Additional file 2: Tab. S1). Further, these ASV contributed to social abilities prediction in $\mathrm{FMT}^{\mathrm{Control}}$ and $\mathrm{FMT}^{p \text {-Cresol }}$ mice, with several ASV affiliated to one uncultured Barnesiella sp., Duncaniella dubosii, and one Anaerobium sp. notably explaining $8 \%, 6 \%$, and $4 \%$ of the score, respectively (Fig. $5 \mathrm{H}$ ). Also, several of these ASV overlapped with the ASV predicting social behavior identified in control and $p$-Cresol-treated mice (Fig. $3 \mathrm{H}$ ). This latter result suggested that specific bacterial taxa could be responsible for social deficits induced by both $p$-Cresol treatment and $\mathrm{FMT}^{p \text {-Cresol }}$. This was not the case for stereotypies (Fig. 5I), which were only modestly explained by microbiota composition post-FMT ${ }^{\text {Control }}$ and $\mathrm{FMT}^{p \text {-Cresol }}$.

In addition, $\mathrm{FMT}^{p-C r e s o l}$ mice exhibited increased fecal levels of $p$-Cresol (Fig. 5J), suggesting that the microbiota of these mice produced more $p$-Cresol compared to the one from FMT ${ }^{\text {Control }}$ mice. Two microbial metabolic pathways are involved in the production of $p$-Cresol from tyrosine. The direct pathway involves the tyrosine lyase ThiH described in Escherichia coli [40] (Additional file 1: Fig. S5A). The indirect pathway involves several enzymes, with the tyrosine aminotransferase TyrB catalyzing the initial step and the $p$ hydroxyphenylacetate decarboxylase Hpd A, B, and C subunits ([30] described in Clostridioides difficile) catalyzing the final step (Additional file 1: Fig. S5A). We therefore searched for proteins with at least 30\% homology to TyrB, ThiH, and $\mathrm{HpdA} / \mathrm{B} / \mathrm{C}$ in bacterial taxa presenting increased abundance in both $p$-Cresoltreated mice and FMT $^{p \text {-Cresol }}$ (Duncaniella dubosii, Barnesiella spp., and Anaerobium spp.). ThiH homologous proteins were present in Duncaniella dubosii, several strains of Barnesiella spp. and in Anaerobium acetethylicum (Table 1, Additional file 4: Tab. S3). Regarding the indirect pathway, we only identified enzymes homologous to HpdA in Duncaniella dubosii and Anaerobium acetethylicum but with generally lower identity scores, and none for TyrB or $\mathrm{HpdB} / \mathrm{C}$ (Table 1, Additional file 4: Tab. S3). This suggested that the direct pathway was likely privileged for $p$-Cresol synthesis in those bacterial species.

To ascertain the bacterial species accounting for $p$ Cresol production, we used RF analysis to identify the ASV counts best predicting fecal $p$-Cresol levels post$\mathrm{FMT}^{\text {Control }}$ and $\mathrm{FMT}^{p \text {-Cresol }}$. Several ASV related to Clostridioides spp. and one Anaerostipes sp. were identified (Additional file 1: Fig. S5B), in line with previous studies which identified relatives of these taxa as bacterial $p$ Cresol producers [30]. In addition, Duncaniella dubosii, one uncultured Barnesiella sp., and several Muribaculaceae spp. contributed to accurately predict $p$-Cresol fecal levels (Additional file 1: Fig. S5B), reinforcing the possible link between $p$-Cresol production and these species overabundant in both $p$-Cresol-treated and $\mathrm{FMT}^{p-\text {-Cresol }}$ mice.

\section{Transplantation of a normal microbiota to $p$-Cresol- treated recipients restores social behavior, VTA dopamine neurons excitability, and fecal $p$-Cresol}

Having shown that $\mathrm{FMT}^{p-\text { Cresol }}$ mice exhibited social behavior deficits, we investigated whether $p$-Cresol-induced behavioral alterations could be restored by FMT $^{\text {Control }}$ (Fig. 6A). While $p$-Cresol-treated mice displayed both social behavior deficits (Fig. $6 \mathrm{~B}-\mathrm{H}$ ) and stereotypies (Fig. 6K, L), as already shown (Fig. 1B-N, Additional file 1: Fig. S3), FMT ${ }^{\text {Control }}$ to $p$-Cresol-treated mice normalized both the number and time spent in social contacts (Fig. 6B, C, E, G, Additional file 1: Fig. S6H, I) and quality (mean duration) of social contacts (Fig. 6D, F) in the dyadic social interaction test. The sociability 


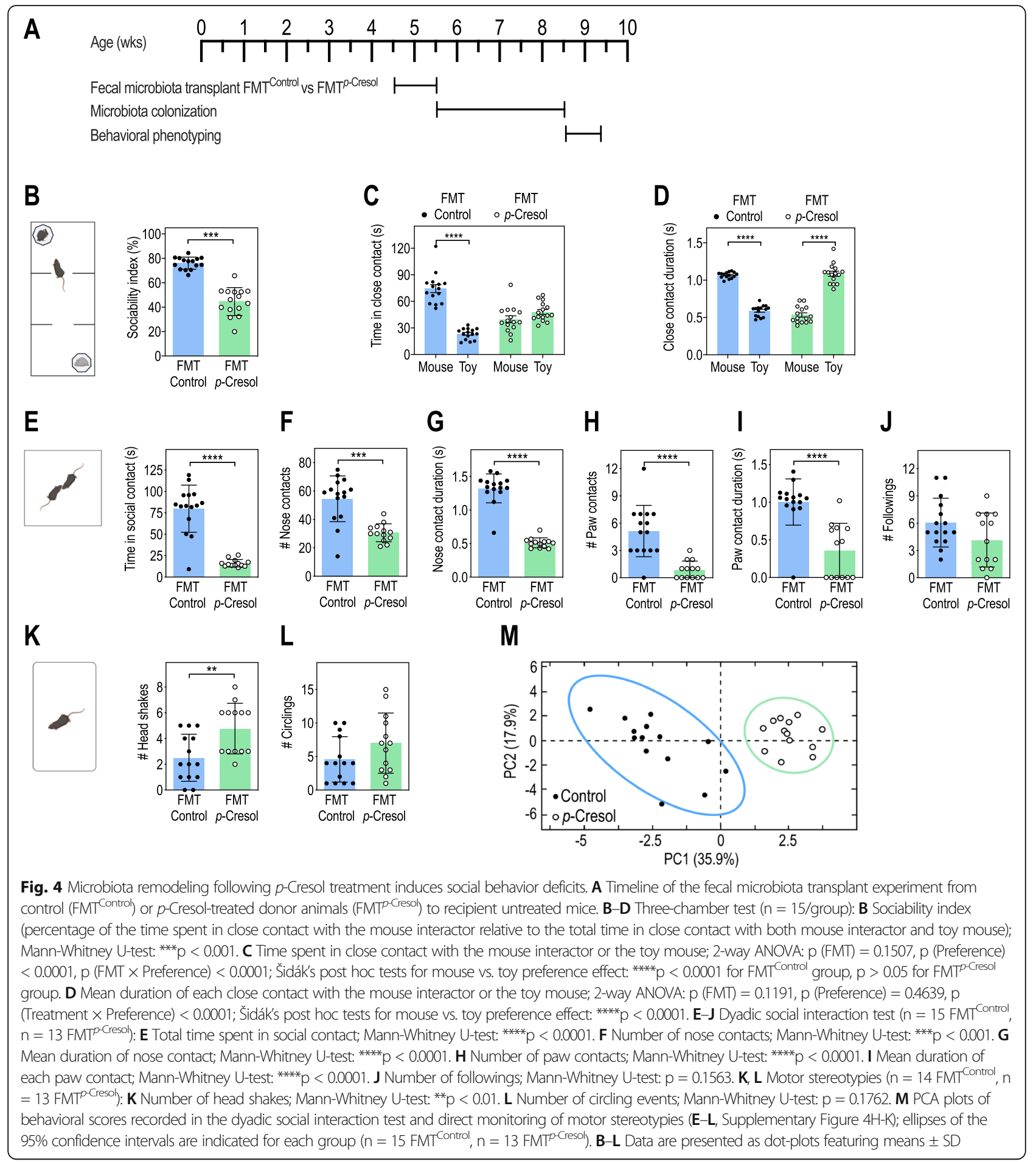

index (Fig. 6H), the preference for the interactor mouse (Fig. 6I, Additional file 1: Fig. S6G), and the quality of social interactions (Fig. 6J) were also rescued, as assessed with the 3-chamber test. While social deficits were fully restored, stereotypies were only partially normalized, as shown by the persistence of circling episodes (Fig. 6K, L,
Additional file 1: Fig. S6J, K). Normalization of $p$-Cresolinduced behaviors by FMT ${ }^{\text {Control }}$ was confirmed by PCA analysis with control mice pre- and post-FMT ${ }^{\mathrm{Control}}$ and $p$-Cresol-treated mice post-FMT ${ }^{\text {Control }}$ clustered on the right side of the $\mathrm{PC} 1$ axis, while $p$-Cresol-treated mice stood alone on the left side (Fig. 6M). Finally, FMT ${ }^{\text {Control }}$ 

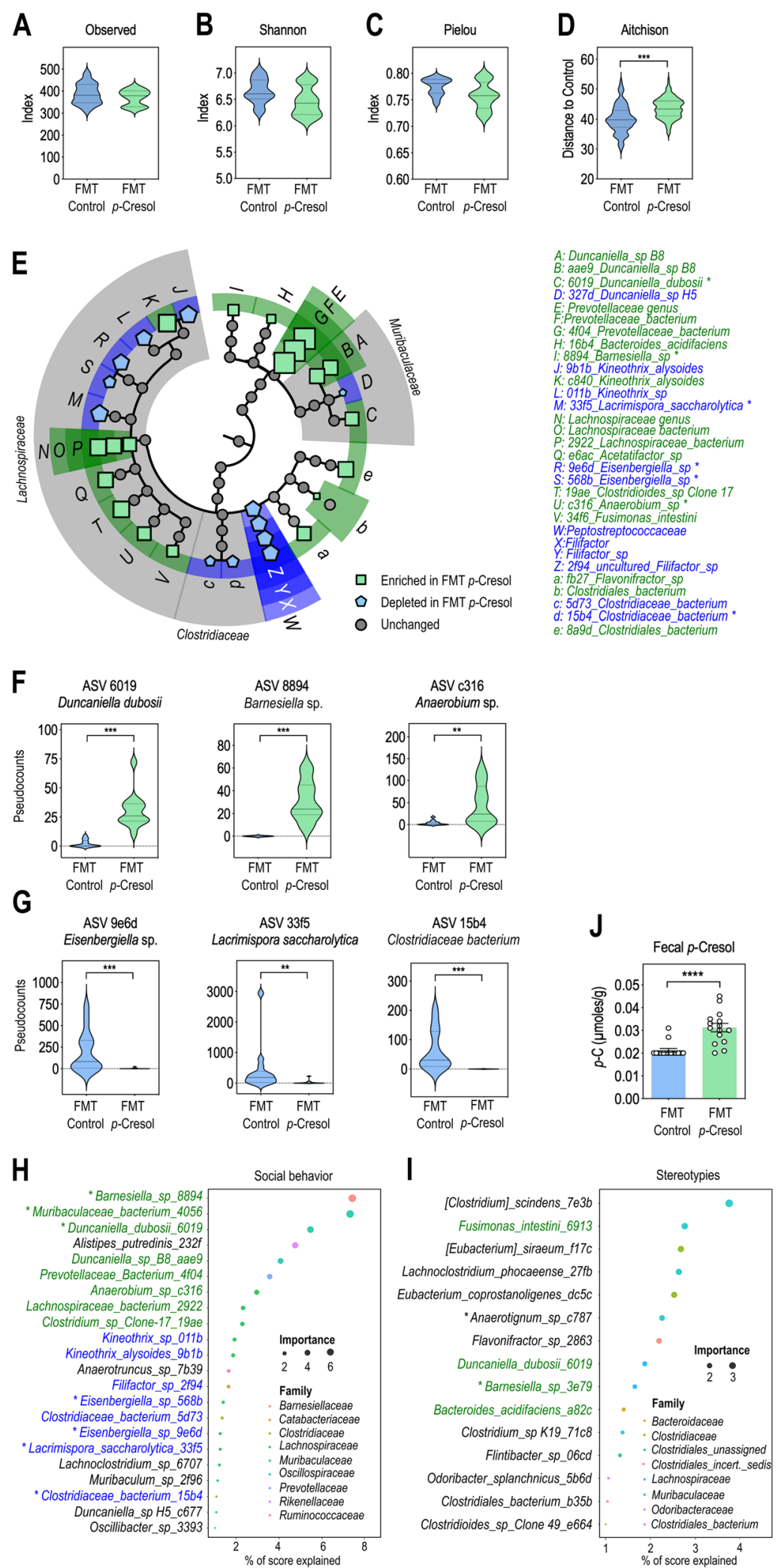

Fig. 5 (See legend on next page.) 
(See figure on previous page.)

Fig. 5 Transplantation of $p$-Cresol-induced microbiota impacts microbial composition, increases $p$-Cresol production and selective bacterial taxa predict social deficits and stereotypies. A-C a-Diversity as measured by observed number of ASV (A), Shannon's (B), and Pielou evenness' (C) indexes ( $n=14 \mathrm{FMT}^{\text {Control }}, \mathrm{n}=12 \mathrm{FMT}^{p \text {-Cresol) }}$; Kruskall-Wallis pairwise test: $p>0.05$. $\mathbf{D} \beta$-diversity as measured by Aitchison distances from control and $p$-Cresol groups based on $16 \mathrm{~S}$ rRNA gene sequencing $\left(n=14 \mathrm{FMT}^{\mathrm{Control}}, \mathrm{n}=12 \mathrm{FMT}^{p \text {-Cresol) }}\right.$. Group differences were tested by pairwise PERM ANOVA, $p=0.001$. E Synthetic cladogram presenting dysregulated ASV and bacterial taxa increased (green) or depleted (blue) upon FMT ${ }^{p \text {-Cresol }}$ identified by ANCOM. Symbol size reflects the value of the corresponding CLR. F, G Pseudocounts plots from selected ASV increased (F) or depleted (G) upon FMT ${ }^{p-C r e s o l}$ identified by ANCOM; Mann-Whitney U-Test: ${ }^{* *} p<0.01$, ${ }^{* * *} p<0.001$. H, I Microbial composition prediction of behavioral scores after FMT ( $n=14 \mathrm{FMT}^{\text {Control }}, \mathrm{n}=12 \mathrm{FMT}^{p \text {-Cresol) }}$. ASV best predicting social interaction deficits $(\mathbf{H})$ and stereotypies $(\mathbf{I})$, as identified by random forest analysis. Only ASV contributing > $1 \%$ accuracy in behavioral scores prediction are presented. ASV identical to or ASV pointing towards the same species as ASV identified by ANCOM as increased or depleted in FMT ${ }^{p \text {-Cresol }}$ as compared to FMT ${ }^{\text {Control }}$ are labeled in green and blue respectively. J Fecal levels of $p$-Cresol 3 weeks post-FMT ( $n=15$ FMT $^{\text {Control }}, \mathrm{n}=15 \mathrm{FMT}^{p \text {-Cresol) }}$; Mann-Whitney U-test: ${ }^{* * * *} \mathrm{p}<$ 0.0001. A-D, F, G Data are presented as violin plots featuring frequency distribution of data, median (dashed line) and quartiles (dotted line). J Data are presented as dot-plots featuring means \pm SD

to $p$-Cresol-treated mice restored both the excitability of VTA dopamine neurons (Fig. $6 \mathrm{~N}, \mathrm{O}$ ) and fecal $p$-Cresol levels (Fig. 6P).

\section{Discussion}

\section{$p$-Cresol selectively induces ASD core behavioral symptoms}

Here, we show that $p$-Cresol-treated mice exhibit social behavior deficits and stereotyped/perseverative behaviors, but no changes in anxiety, locomotion, or cognition. This suggests a possible causal relationship between elevated $p$-Cresol levels and ASD core symptoms. While several other metabolites modified behavior when administered to rodents, none of them selectively induced ASD core symptoms. For example, the SCFA propionate did induce not only social interaction deficits and stereotypies, but also anxiety, hyperlocomotion, and cognitive deficits [17]. Indoles increased social contacts and anxiety, reduced locomotor activity in rats [41], and exacerbated emotional behaviors in chronically stressed mice [42]. 4-EPS increased anxiety and startle reflex in mice but had no impact on social behavior or stereotypies [20]. Frequent ASD comorbidities are hyperactivity,
ID, and anxiety disorder [4] and we show that related behavioral domains (locomotor activity, cognition, anxiety) are not impacted by $p$-Cresol exposure in mice. It appears that propionate, indoles, or 4-EPS induce anxiety, which could interfere with social and cognitive abilities and explain their broader effects. These data collectively suggest that microbial metabolites likely interfere with several dimensions of behavior impacted in ASD, each with its specificities, with $p$-Cresol selectively impacting social behavior and stereotyped/perseverative behaviors, related to ASD core symptoms.

While previous studies in ASD patients have shown increased urinary and fecal levels of $p$-Cresol, we still do not know whether circulating levels of $p$-Cresol are increased in ASD patients as $p$-Cresol has not been measured in blood to the best of our knowledge. One recent study in the BTBR model of idiopathic ASD showed that acute intravenous (i.v.) $p$-Cresol injection exacerbated anxiety, hyperactivity, stereotypies, and reduced social preference [43]. Compared to the selective effects of $p$ Cresol in our model, these broader effects may be explained by differences in genetic background (BTBR versus $\mathrm{C} 57 \mathrm{BL} / 6)$ or the mode of $p$-Cresol administration

Table 1 Predicted proteins homologous to enzymes involved in metabolic pathways from tyrosine to $p$-Cresol in species related to bacterial taxa identified as upregulated both upon $p$-Cresol treatment and upon FMT ${ }^{\text {-Cresol }}$

\begin{tabular}{|c|c|c|c|c|c|c|}
\hline \multirow[t]{3}{*}{ Species } & \multicolumn{3}{|l|}{ Direct pathway } & \multicolumn{3}{|l|}{ Indirect pathway } \\
\hline & \multicolumn{3}{|l|}{ ThiH } & \multicolumn{3}{|l|}{ HpdA } \\
\hline & Accession & $\%$ Identity & E-score & Accession & $\%$ Identity & E-score \\
\hline Anaerobium acetethylicum & WP_091230597.1 & 38.90 & $3.00 E-72$ & WP_091236185.1 & 37.62 & $4 E-66$ \\
\hline Duncaniella dubosii & WP_123615573.1 & 46.85 & $3.00 E-121$ & WP_123485485.1 & 32.59 & $5 E-13$ \\
\hline Barnesiella sp. & MBD5257534.1 & 48.49 & $1.00 E-129$ & - & - & - \\
\hline Barnesiella sp. An55 & WP_087424377.1 & 49.04 & $3.00 E-125$ & - & - & - \\
\hline Barnesiella intestinihominis & WP_195342987.1 & 48.65 & $2.00 E-124$ & - & - & - \\
\hline Barnesiella viscericola & WP_025277286.1 & 47.95 & $6.00 E-123$ & - & - & - \\
\hline
\end{tabular}

Reference enzyme protein sequences from ThiH (NP 418417.1, 2-iminoacetate synthase from Escherichia coli K12, metabolizing tyrosine to $p$-Cresol) and HpdA (sp|Q84F14|HPDA_CLODI, 4-hydroxyphenylacetate decarboxylase activating enzyme from Clostridioides difficile, metabolizing 4-hydroxyphenylacteate to $p$-Cresol) were used to conduct a Blast search against sequences from the Anaerobium genus and from the Muribaculaceae family. Only sequences covering more than $50 \%$ of the query sequence and presenting amino acid identity above $30 \%$ were considered potential homologous enzymes. See also Tab. S3 (Additional file 4 ) for the full blast analysis 


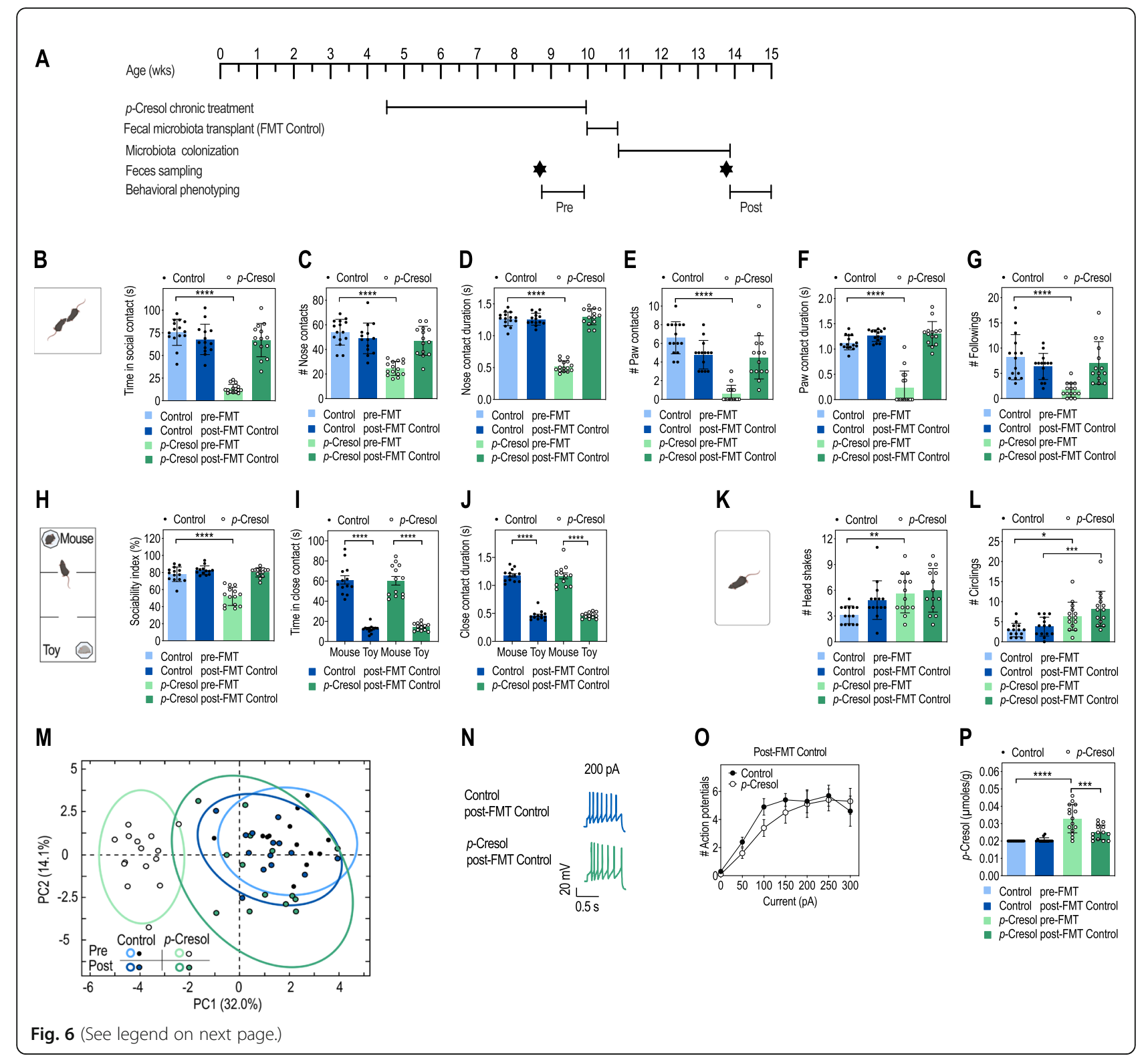


(See figure on previous page.)

Fig. 6 Transplantation of microbiota from control mice to $p$-Cresol-treated mice restores social behavior deficits, VTA dopamine neurons excitability, and fecal $p$-Cresol levels. A Timeline of the transplantation procedure with fecal microbiota from control donor mice (FMT ${ }^{\text {Control) }}$ to control or $p$-Cresol-treated recipient mice. B-G Dyadic social interaction test pre-FMT ${ }^{\text {Control }}$ and 3 weeks post-FMT ${ }^{\text {Control }}$ ( $\mathrm{n}=15 / \mathrm{group}$ pre-FMT, $\mathrm{n}$ = 14/group post-FMT): $\mathbf{B}$ Total time spent in social contact; 2-way ANOVA: $\mathrm{p}$ (Treatment) $<0.0001, \mathrm{p}\left(\right.$ FMT $\left.{ }^{\text {Control}}\right)<0.0001, \mathrm{p}$ (Treatment $\times$ FMT ${ }^{\text {Control })}<0.0001$; Šidák's post hoc tests for treatment effect: ${ }^{* * *} p<0.0001$ for pre-FMT groups, $p>0.05$ for post-FMT groups. C Number of nose contacts; 2-way ANOVA: $\mathrm{p}$ (Treatment) $<0.0001, \mathrm{p}\left(\mathrm{FMT}^{\mathrm{Control}}\right)=0.0022, \mathrm{p}$ (Treatment $\left.\times \mathrm{FMT}^{\mathrm{Control}}\right)<0.0001$; Šidák's post hoc tests for treatment effect: ${ }^{* * * *} p<0.0001$ for pre-FMT groups, $p>0.05$ for post-FMT groups. D Mean duration of each nose contact; 2 -way ANOVA: $p$ (Treatment) $<0.0001, \mathrm{p}\left(\mathrm{FMT}^{\text {Control }}\right)<0.0001, \mathrm{p}$ (Treatment $\times \mathrm{FMT}^{\text {Control }}$ ) $<0.0001$; Šidák's post hoc tests for treatment effect: ${ }^{* * * *} \mathrm{p}<0.0001$ for pre-FMT groups, $p>0.05$ for post-FMT groups. E Number of paw contacts; 2-way ANOVA: $p$ (Treatment) $<0.0001, p\left(\right.$ FMT $\left.{ }^{\text {Control }}\right)=0.0221, p$ $\left(\right.$ Treatment $\left.\times \mathrm{FMT}^{\text {Control }}\right)<0.0001$; Šidák's post hoc tests for treatment effect: ${ }^{* * *} \mathrm{p}<0.0001$ for pre-FMT groups, $\mathrm{p}>0.05$ for post-FMT groups. $\mathbf{F}$ Mean duration of each paw contact; 2-way ANOVA: $\mathrm{p}$ (Treatment) $<0.0001, \mathrm{p}\left(\mathrm{FMT}^{\text {Control}}\right)<0.0001, \mathrm{p}$ (Treatment $\left.\times \mathrm{FMT}^{\text {Control }}\right)<0.0001$; Šidák's post hoc tests for treatment effect: ${ }^{* * *} p<0.0001$ for pre-FMT groups, $p>0.05$ for post-FMT groups. G Number of followings; 2 -way ANOVA: $p$ $($ Treatment $)=0.0020, p\left(F M T^{\text {Control }}\right)=0.0516, p\left(\right.$ Treatment $\left.\times F T^{\text {Control }}\right)=0.0002$; Šidák's post hoc tests for treatment effect: ${ }^{* * * *} p<0.0001$ for pre-FMT groups, $\mathrm{p}>0.05$ for post-FMT groups. H-J Three-chamber test pre-FMT ${ }^{\text {Control }}$ and 3 weeks post-FMT ${ }^{\text {Control }}(\mathrm{n}=15 / \mathrm{group}$ pre-FMT, $\mathrm{n}=$ 14/group post-FMT): $\mathbf{H}$ Sociability index pre-FMT ${ }^{\text {Control }}$ and 3 weeks post-FMT ${ }^{\text {Control; }}$ 2-way ANOVA: $p$ (Treatment) $=0.0004, p\left(F M T^{\text {Control }}\right.$ ) $<0.0001$, $p\left(\right.$ Treatment $\left.\times F M^{\text {Control }}\right)=0.0032$; Šidák's post hoc tests for treatment effect: ${ }^{* * *} p<0.0001$ for pre-FMT groups, $p>0.05$ for post-FMT groups. I Time in close contact with the mouse interactor or the toy mouse post-FMT ${ }^{\text {Control }}$; 2 -way ANOVA: $p$ (Treatment) $=0.8480, p$ (Preference) $<$ 0.0001, $\mathrm{p}$ (Treatment $\times$ Preference $)=0.6498$; Šidák's post hoc tests for mouse vs. toy preference: ${ }^{* * * *} p<0.0001$. J Mean duration of each nose contact with the mouse interactor or toy mouse post-FMT Control; 2-way ANOVA: $\mathrm{p}$ (Treatment) $=0.9067, \mathrm{p}$ (Preference) $<0.0001, \mathrm{p}$ (Treatment $x$ Preference $)=0.8713$; Šidák's post hoc tests for mouse vs. toy preference: ${ }^{* * *} \mathrm{p}<0.0001$. K, L Motor stereotypies pre-FMT Control and 3 weeks post$\mathrm{FMT}^{\text {Control }}\left(\mathrm{n}=15\right.$ /group pre-FMT, $\mathrm{n}=15$ /group post-FMT): $\mathbf{K}$ Number of head shakes; 2 -way ANOVA: $\mathrm{p}$ (Treatment) $=0.0021, p\left(\mathrm{FMT} T^{\text {Control }}\right)=$ 0.0715, $p\left(\right.$ Treatment $\times$ FMT $\left.^{\text {Control }}\right)=0.2334$; Šidák's post hoc tests for treatment effect: ${ }^{*} p<0.01$ for pre-FMT groups, $p>0.05$ for post-FMT groups. L Number of circling events; 2-way ANOVA: $\mathrm{p}$ (Treatment) $<0.0001, \mathrm{p}\left(\mathrm{FMT}^{\text {Control }}\right)=0.1133, \mathrm{p}\left(\right.$ Treatment $\left.^{\mathrm{P}} \times \mathrm{FMT}^{\text {Control }}\right)=0.5555$; Šidák's post hoc tests for treatment effect: ${ }^{*} p<0.05,{ }^{* * *} p<0.001$. M PCA plots of behavioral scores recorded in the dyadic social interaction test and direct monitoring of motor stereotypies pre-FMT ${ }^{\text {Control }}$ and 3 weeks post-FMT ${ }^{\text {Control }}$ (B-G, K, L, Additional file 1: Fig. S6H-K); ellipses of the $95 \%$ confidence intervals are indicated for each group ( $n=15 /$ group pre-FMT, $n=14 /$ group post-FMT). N , O Electrophysiological recordings of dopamine neurons activity in the VTA 3 weeks post-FMT ${ }^{\text {Control: }} \mathbf{N}$ Representative traces of dopamine neurons activity in patch-clamp experiments performed post-FMT ${ }^{\text {Control }}$ after a 200-pA current injection. O Number of action potentials evoked by different current injection steps ( $n=3$ animals/group, 10 cells recorded/animal); 2-way ANOVA: $p$ (Treatment) $=0.5474, p$ (Current) $<0.0001, p$ (Treatment $\times$ Current) $=0.3640 ;$ Šidák's post hoc tests for treatment effect: $p>0.05$. P Fecal levels of $p$-Cresol pre-FMT ${ }^{\text {Control }}$ and 3 weeks post-FMT ${ }^{\text {Control }}(n=15 / g$ roup pre-FMT, $n=14$ /

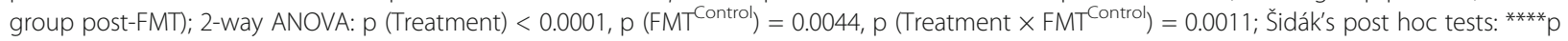
$<0.0001 ;{ }^{* * *} \mathrm{P}<0.001$. B-L, P Data are presented as dot-plots featuring means \pm SD. $\mathbf{O}$ Data are presented as means \pm SD

(i.v. versus oral, acute versus chronic). Since primary exposure to microbial metabolites occurs in the GI tract, the per os delivery of $p$-Cresol that we used reflects more closely than i.v. GI exposure to endogenous $p$-Cresol produced by the microbiota. We have shown that this does not lead to elevated circulating $p$-Cresol in serum, suggesting limited systemic effects compared to i.v. injection, and possibly explaining the selective induction of social deficits and stereotyped/perseverative behaviors.

\section{p-Cresol induces social behavior deficits via microbiota remodeling}

Three independent meta-analyses reported bacterial $\beta$ diversity changes associated with increased abundance of the genus Clostridioides and decreased abundance of the genus Bifidobacterium in ASD patients as compared to neurotypical controls [8-10]. While we observed $\beta$ diversity changes in $p$-Cresol-treated mice, Clostridioides and Bifidobacterium were not impacted at the genus level. Instead, we observed selective changes in lower taxonomic levels (mostly ASV, species). Confounding factors (diet, treatment, sex, age) have not been considered in most human microbiota studies, possibly leading to discrepancies in the identified changes [8-10]. Also, the human and murine microbiota differ in terms of bacterial species representation. Indeed, we highlighted selective changes in murine-specific bacteria upon $p$ Cresol exposure. Notably Muribaculaceae and Turicimonas are both dominant taxa in the murine microbiota [44]. While changes in $\beta$-diversity have been observed in ASD murine models [20-24], the lower taxonomic resolution of most studies precludes adequate comparison with our findings in $p$-Cresol-treated mice. However, it is worth mentioning that mouse and human microbiota share $95.2 \%$ of metabolic functions [45]; we therefore posit that studying $p$-Cresol, which is a product from microbial metabolism common to murine and human microbiota, reinforces the translationality of our findings. Furthermore, in our experimental paradigm, we achieve effect sizes for the increase in urinary and fecal $p$-Cresol (4-fold and 1.65-fold, respectively) within the range of the changes observed in ASD patients (1.3 to 3.4-fold for urinary $p$-Cresol $[14,15,28], 1.23$ to 1.41 fold for fecal $p$-Cresol $[18,19,27])$. This strengthens the translational relevance of findings and suggests that $p$ Cresol could contribute to ASD core symptoms in humans. 
Two metabolic pathways have been described for $p$-Cresol production from tyrosine. The direct pathway described in $E$. coli involves $\mathrm{ThiH}$ [40], while the indirect pathway present in C. difficile and Blautia hydrogenotrophica involves TyrA/B/C and Hpd [30]. These enzymes have not yet been formally described in Duncaniella dubosii, Barnesiella spp., or Anaerobium spp. (which are upregulated in $p$-Cresol-treated and FMT $^{p \text {-Cresol }}$ mice), but ThiH homologous proteins are predicted in these species, suggesting that they can indeed produce $p$-Cresol via the direct pathway. This is reinforced by the fact that the counts of ASV related to Duncaniella dubosii, one Barnesiella sp., and several Muribaculaceae bacteria predict fecal $p$-Cresol levels in $\mathrm{FMT}^{p \text {-Cresol }}$ mice. Conversely, FMT $^{\text {Control }}$ to $p$-Cresol-treated mice decreased $p$-Cresol levels. This latter result suggested that the capacity to metabolize $p$-Cresol was reduced after FMT ${ }^{\text {Control }}$, possibly explaining the rescue of both social behavior and VTA dopamine neurons excitability. Finally, the fact that $\mathrm{FMT}^{p \text {-Cresol }}$ and FMT ${ }^{\text {Control }}$ respectively induce or rescue social deficits and, to a lesser extent, stereotypies, argues for a strong contribution of the $p$-Cresol-induced changes in microbial composition to social behavior in our model. Future metagenomic and metabolomic studies will help refine the bacterial species and metabolic pathways linking $p$-Cresol to social behavior deficits. Also, future experiments conducted in germ-free animals would allow to determine the critical contribution of microbiota remodeling induced by $p$-Cresol to the induction of ASD-like behaviors. Finally, addressing whether $p$-Cresol is dysregulated in ASD genetic and environmental models could allow for further demonstration of its pathophysiological relevance.

\section{Exposure to $p$-Cresol impacts central dopamine neurons activity}

Social interactions are pleasurable events for humans and animals, as shown by the activation of the reward circuit by social stimuli. This activation is blunted in ASD [46]. The VTA is a key subcortical node of the mesolimbic reward pathway [47] and ASD patients display defects in VTA connectivity [33]. Moreover, blockade of dopamine neurons in the lateral VTA reduced social interactions in rodents [32, 48]. Further, impaired VTA dopamine neurons activity was observed in both environmental (MIA, DIO [21, 37]) and genetic (Shank3b-, Nlgn3-, and Ube3a-deficient mice [22, 3436]) mouse models of ASD. Microbiota manipulations normalize both social behavior and VTA dopamine neurons activity in Shank3b-deficient and DIO ASD models $[21,22]$, as well as in $p$-Cresol-treated mice (this study). Taken together, these results support a model in which $p$-Cresol-induced changes in microbial composition impair VTA dopamine neurons activity. As dopamine neurons in the lateral VTA project to the nucleus accumbens medial shell as part of a "socially engaged reward circuit" [32], we posit that disruption of dopamine in the reward circuit contributes to the social behavior deficits induced by $p$-Cresol. Our data are in agreement with a recent study showing that acute intravenous administration of $p$-Cresol increased dopamine turnover in several areas of the reward circuit in the BTBR ASD model [43]. How $p$-Cresol-induced microbiota changes impact VTA activity, central dopamine and the social reward circuit remains to be further investigated.

As for stereotyped behaviors, their induction appears independent on the reduced excitability of dopamine neurons in the lateral VTA, as stereotypies are only partially rescued by FMT ${ }^{\text {Control }}$, while social behaviors and VTA activity are. One possible pathway could be through dopamine nigrostriatal circuits that are central to the expression of stereotypies $[49,50]$. Still, unbalanced dopamine circuits would be at stake, reinforcing the notion that $p$-Cresol could generally contribute to alter central dopamine balance.

\section{Possible underlying mechanisms}

Some microbial metabolites are ligands to host receptors: 3-indoxylsulfate binds to the aryl-hydrocarbon receptor [51], indole-3-propionate to the xenobiotic sensor pregnane $X$ receptor [52], and propionate to GPR41 and GPR43 [53]. Yet their signaling role has been mainly investigated in metabolic, GI, or autoimmune disorders [51-53], and not in ASD. Indoles, SCFA, and their receptors are detected in the brain suggesting that these metabolites could act centrally [26, 54, 55]. However, this hypothesis remains to be tested. While both urinary and fecal $p$-Cresol levels are increased in $p$-Cresoltreated mice, serum levels are not, suggesting that $p$ Cresol does not reach bioactive levels in the brain and does not act centrally per se. Although $p$-Cresol is produced by bacterial metabolism of dietary tyrosine in the colonic lumen, limited amounts of $p$-Cresol actually reach the general circulation, as $p$-Cresol is rapidly uptaken by colonocytes and detoxified by conjugation into $p$ Cresylsulfate $[56,57]$. Most of the remaining circulating fraction of free $p$-Cresol is also conjugated by hepatocytes into $p$-Cresylsulfate and $p$-Cresylglucuronide and excreted in the urine $[56,57]$. This may explain why we do not observe elevated serum levels of $p$-Cresol in our mice. It remains an open question whether $p$-Cresylsulfate and $p$-Cresylglucuronide could relay centrally the effects of $p$-Cresol.

Alternately, if $p$-Cresol does not act directly on the brain as suggested by our findings, $p$-Cresol could act locally in the gut. Should this be the case, both the yet-tobe-identified signaling properties of $p$-Cresol or bacterial signals from the microbiota induced by $p$-Cresol could be at stake. These signals could target gut immune and 
neuroendocrine cells, enteric neurons, or vagal afferences, resulting in perturbations of the microbiota-gutbrain axis and altered behavior $[1,26]$. The vagus nerve appears as a route of choice for bacteria to communicate with the brain and influence social behavior [58]. In support of this, vagotomy experiments showed that the beneficial effects of the probiotic Lactobacillus reuteri on social behavior in the Shank3b-KO ASD mouse model were relayed by the vagus [22]. Furthermore, mounting evidence support the fact that the microbiota can influence dopamine circuits [59] and that vagal sensory neurons in the gut are critical for central dopamine release [60]. Whether and how the vagus contributes to relay the effects of $p$-Cresol-induced microbiota remodeling on dopamine circuits and social behavior remains to be investigated.

\section{Conclusions and perspectives}

We found that $p$-Cresol-treated mice exhibited social interaction deficits and stereotypies, reminiscent of ASD core symptoms in humans. Social behavior deficits were dependent on changes in microbiota composition and were associated with reduced excitability of VTA dopamine neurons as well as elevated $p$-Cresol production. The mechanisms underlying the induction of stereotypies by $p$-Cresol remain to be determined. Because $p$ Cresol levels are elevated in ASD patients, our study suggests that increased levels of this metabolite could contribute to social behavior deficits in ASD. Further, the ability of a control microbiota to normalize $p$-Cresol levels, VTA dopamine neurons excitability, and social behavior when transplanted to $p$-Cresol-treated mice provide a rationale for clinical trials aimed at studying the beneficial impact of microbiota interventions targeting $p$-Cresol production to alleviate core social deficits in ASD.

\section{Methods}

\section{Animals}

Weaned C57BL/6J male mice (21 to 28 day-old) were ordered to Charles River (France). Since the sex ratio for ASD is biased towards 3 males diagnosed for 1 female suggesting a higher susceptibility of males [61], only males were considered in this study. Animals were randomly assigned to experimental groups and housed in medium-size (up to 5 animals) open cages filled with wooden bedding, one plastic house and nesting material for enrichment, in a temperature- $\left(22-24{ }^{\circ} \mathrm{C}\right)$ and hygrometry- (70-80\%) controlled room, under 12 h lightdark cycle (8:00 a.m.-8:00 p.m.). Mice had ad libitum access to water and standard chow (reference 4RF25, Mucedola).

\section{p-Cresol treatment}

Mice were habituated for 6 days to our animal facility before starting treatment with $p$-Cresol (reference W233706-SAMPLE-K, Sigma-Aldrich) dispensed in sterile drinking water at a concentration of $0.25 \mathrm{~g} / \mathrm{L}$. Bottles were renewed twice weekly. This amounts to a dose of $50 \mathrm{mg} / \mathrm{Kg} / 24 \mathrm{~h}$ based on a mean body mass of $25 \mathrm{~g}$ and a mean drinking water consumption of $5 \mathrm{~mL} / 24 \mathrm{~h}$. Body weight, consumption of water, and chow were monitored weekly.

\section{Quantification of $p$-Cresol in urine and serum by gas chromatography coupled to mass spectrometry (GC-MS)}

Mice were individually placed in a clean empty plastic cage and urine was collected over $10 \mathrm{~min}$ and centrifuged $(12,000 \times g, 5 \mathrm{~min})$. The supernatant was snappedfrozen in liquid nitrogen and stored at $-80{ }^{\circ} \mathrm{C}$ until analysis. Blood was collected by cardiac puncture, transferred in Eppendorf tubes, and allowed to clot on bench RT for $30 \mathrm{~min}$. After centrifugation $(12,000 \times g, 10 \mathrm{~min}$, RT), serum was collected, snapped-frozen in liquid nitrogen, and stored at $-80{ }^{\circ} \mathrm{C}$ until analysis. Prior to gas chromatography-mass spectrometry (GC-MS), urine (20 $\mu \mathrm{L})$, and serum $(100 \mu \mathrm{L})$ samples were prepared as follows: (i) samples were spiked with $10 \mu \mathrm{L}$ internal standard solution (myristic acid- $\mathrm{d}_{27}$ in isopropanol, $750 \mathrm{mg} /$ $\mathrm{mL}$ ), (ii) $850 \mu \mathrm{L}$ of ice-cold methanol were added, followed by centrifugation for $20 \mathrm{~min}\left(4{ }^{\circ} \mathrm{C}, 16,000 \times g\right)$, (iii) $750 \mu \mathrm{L}$ of supernatants were transferred to silanized dark $2 \mathrm{~mL}$ autosampler vials and evaporated to dryness in a rotational vacuum concentrator $\left(45^{\circ} \mathrm{C}, 20 \mathrm{mbar}, 2\right.$ h), (iv) $50 \mu \mathrm{L}$ of methoxyamine solution ( $2 \%$ in pyridine) were added and the samples were incubated overnight at $\mathrm{RT}$, and (v) $100 \mu \mathrm{L}$ of N-methyl-trimethylsilyl-trifluoroacetamide (MSTFA) containing 1\% of trimethylchlorosilane (TMCS) solution were added; the samples were incubated at $60{ }^{\circ} \mathrm{C}$ for $1 \mathrm{~h}$ and transferred to dark autosampler vials with $250-\mu \mathrm{L}$ silanized inserts. The detailed instrumental conditions for GC-MS analysis were based on a previous study [62], with minor modifications to quantify $p$-Cresol. Briefly, the samples were analyzed in an Agilent 7890B-5977B Inert Plus GC-MS system. Two microliters of each sample were injected in a split inlet (1:10 ratio). The chromatographic column was an Agilent ZORBAX DB5- MS (30 $\mathrm{m} \times 250 \mu \mathrm{m} \times 0.25 \mu \mathrm{m}+$ $10 \mathrm{~m}$, Duraguard). The temperature gradient was 37.5 min long and the mass analyzer was operated in full scan mode between 50 and $600 \mathrm{~m} / \mathrm{z}$. Blank and pooled quality control (QC) samples in several dilutions were included in the analysis to ensure that the measurements were reproducible. There were no external contaminations and the monitored analytes were responding linearly to the detector. 


\section{Quantification of $p$-Cresol in fecal samples by liquid} chromatography-tandem mass spectrometry (LC-MS/MS) Mice were individually placed in a clean empty plastic cage and feces were collected over $10 \mathrm{~min}$ and immediately snapped-frozen in liquid nitrogen and stored at $80{ }^{\circ} \mathrm{C}$ until analysis. Thawed fecal pellets were extracted as previously described in [63]. Briefly fecal pellets were homogenized in ice-cold water (1:1 w:v ratio), vortexed $30 \mathrm{~s}$, ultrasonicated for $2 \mathrm{~min}$, then subjected to ultracentrifugation $\left(175,000 \times g, 30 \mathrm{~min}, 4{ }^{\circ} \mathrm{C}\right)$. For the determination of unconjugated $p$-Cresol by LC-MS/MS, fecal supernatant preparation included addition of an internal standard ( $1 \mathrm{ng} / \mathrm{mL}$ of $p$-Cresol-d8, Eurisotop, St-Aubin, France) and derivatization with dansyl chloride according to [64]. Dansylation was shown to improve signal intensity in LC-MS/MS analysis of low-abundance phenolic compounds [65]. Briefly, $100 \mu \mathrm{L}$ of fecal supernatant were mixed with $300 \mu \mathrm{L}$ of an acetonitrile solution vortexed, incubated at $-20{ }^{\circ} \mathrm{C}$ for $20 \mathrm{~min}$ and centrifuged $\left(12,000 \times g, 7 \mathrm{~min}, 4{ }^{\circ} \mathrm{C}\right)$. Two hundred microliters of the resulting supernatant were mixed with $50 \mu \mathrm{L}$ of $0.1 \mathrm{M}$ carbonate-bicarbonate solution $(\mathrm{pH}=$ 10), $125 \mu \mathrm{L}$ of water, and $125 \mu \mathrm{L}$ of dansyl chloride at a final concentration of $0.5 \mathrm{mg} / \mathrm{mL}$. After vortexing, the mixture was incubated for $10 \mathrm{~min}$ at $60{ }^{\circ} \mathrm{C}$ and extracted with $2.5 \mathrm{~mL}$ of hexane. Hexane residues were air-dried and reconstituted with $1 \mathrm{~mL}$ of acetonitrile-water (1:1, $\mathrm{v} / \mathrm{v})$. Fifteen microliters of sample were injected and analyzed using a Waters ACQUITY ultraperformance liquid chromatography (UPLC) system equipped with a binary solvent delivery manager and sample manager (Waters Corporation, Milford, MA, USA) and coupled to a tandem quadrupole-time-of-flight (Q-TOF) mass spectrometer equipped with an electrospray interface (Waters Corporation). Quantifications were performed by referencing calibration curves obtained with internal standards. Unconjugated $p$-Cresol was identified by comparing with the accurate mass and the retention time of the reference standard ( $p$-Cresol, Sigma-Aldrich) in our in-house library.

\section{Behavioral testing}

Behavioral phenotyping started after a 4-week treatment with $p$-Cresol and lasted for 1-2 weeks during which treatment was continued. All behavioral experiments were performed during the day. The animals followed multiple behavioral tests respecting their resting hours, with a minimum of 2 days between tests. Standard behavioral tests that have extensively been used to characterize genetic and environmental models of ASD [66-70] were implemented to assess the behavioral dimensions impacted in ASD. Tab. S5 (Additional file 1) recapitulates the behavioral tests performed in the course of this study.

\section{Social abilities}

Three-chamber sociability test The stimulus mouse was habituated to the cylindrical cages (20-min sessions, repeated 3 days before the testing day). The apparatus consisted in a rectangular non-transparent plexiglass box $(60 \times 30 \mathrm{~cm})$, divided in three compartments (object chamber, empty chamber, and unfamiliar mouse chamber) connected by open doors (4-cm width) to allow the mouse to move freely between the different compartments. The unfamiliar mouse or the toy mouse were placed in wired cylindrical cages. The test consisted in a habituation phase to the empty apparatus only in the presence of empty cylindrical cages during $5 \mathrm{~min}$ and a sociability phase where the unfamiliar mouse or the toy mouse is introduced in the cylindrical cages. Time spent in the 2 chambers were video-tracked over $10 \mathrm{~min}$ and analyzed a posteriori during the habituation and sociability phase using the ANY-maze software. The number and the time spent in close contact with each of the empty cylindrical cage (during the habituation phase) as well as with the stimulus mouse or with the toy mouse contained in wired cylindrical cage (during the sociability phase) were manually scored by an experienced experimenter blind to the experimental group. The mean duration of close contacts was calculated from these data $[67,71-73]$. The relative position of stimulus unknown mouse (versus mouse toy) was counterbalanced between groups. Sociability was evaluated by calculating the interaction ratio, i.e., the percentage of time spent in close contact with the wire cage containing the mouse interactor relative to the total time spent in close contact with the wire cage containing the mouse interactor or the toy mouse (test phase). There was no bias in chamber preference, empty cylindrical wire cage preference, or interaction ratio during the habituation phase (Additional file 1: Fig. S2A-E, Fig. S4A-E, Fig. S6A-E).

Dyadic social interactions Direct social interactions were recorded in open-field arena with a low light intensity (15 Lux). The subject mouse was put in presence with un unfamiliar sex- and age-matched interactor and their interaction was recorded for $10 \mathrm{~min}$. Manual scoring by an experienced experimenter blind to the experimental group was performed a posteriori by recording time spent in social contact events, number of nose and paw contacts, time spent in nose and paw contact, number and time spent in self-grooming, number of selfgrooming events after social contact, number of rearing and grooming events, and number of circling episodes. The mean duration of nose and paw contacts was calculated from these data [67, 71-73]. 


\section{Stereotyped/perseverative behaviors}

Motor stereotypies The subject mouse was placed in a clean standard home cage $(21 \times 11 \times 17 \mathrm{~cm})$ covered with a thick layer of fresh sawdust $(4 \mathrm{~cm})$ and recorded for 10 min with light intensity set at 40 Lux. Manual scoring by an experienced experimenter blind to the experimental group was performed a posteriori by computing the number of events of head shake, rearing, digging, grooming, circling episodes, and total time spent digging and grooming [66].

Marble burying test Marble burying was used as a measure of perseverative behavior [74]. The subject mouse was placed in a clean standard home cage $(21 \times 11 \times 17 \mathrm{~cm})$ filled with $4 \mathrm{~cm}$ of fresh sawdust on which 20 glass marbles (diameter $1.5 \mathrm{~cm}$ ) were disposed with light intensity set at 40 Lux. The number of marbles buried (more than half of its surface outside of sawdust) during a 30-min session was monitored by an experienced experimenter blind to the experimental group [66].

Y-maze spontaneous alternation task Spontaneous alternation behavior was used to assess behavioral flexibility and perseveration [75-77]. The apparatus consisted in three arms made of plexiglass $(40 \times 9 \times 16 \mathrm{~cm})$ containing different symbols to be differentiated. We placed the mice in the center and allowed it to explore the arms for 5 min with a low light intensity of 15 Lux. We measured the willingness of mice to explore new environments and we scored different patterns as spontaneous alternation (SPA), alternate arm returns (AAR), and same-arm return (SAR). We then calculated the percentage of SPA, AAR, and SAR [66, 78].

\section{Locomotor activity}

Actimetry The measurement of individual spontaneous activity was performed in actimetry chambers (Imetronic) consisting of cages equipped with infrared beams able to detect in real time horizontal and vertical movements (rearing events). Animals were individually placed in actimetry chambers under a 12-h light/dark cycle, with free access to food and drinking water. To avoid biases in measurements due to stress possibly inducing hyperlocomotion in a novel environment, a habituation period (day 0, 11:00 p.m.-day 1, 8:00 a.m.) preceded the 24-h recording of horizontal activity (day 1, 8:00 a.m.day 2, 8:00 a.m.) [68].

\section{Anxiety}

Open-field test Mice were individually placed in the corner of a white and opaque quadratic arena $(40 \times 40$ $\mathrm{cm}$ ) and allowed to explore for $10 \mathrm{~min}$ under low illumination (15 Lux). Total distance traveled, time spent in center and number center entries were analyzed by videotracking using the ANY-Maze software $[68,69]$.

Novelty-suppressed feeding test Mice were fooddeprived for $16 \mathrm{~h}$, with unlimited access to drinking water. A pellet of food was positioned in the center of an open field covered with $1 \mathrm{~cm}$ of sawdust with light intensity set at 60 Lux. The subject mouse was then introduced in a corner of the arena and the latency (s) for the first bite in the pellet recorded. Immediately after the test, the animal was placed in a clean cage with ad libitum access to food and water [66, 78].

Zero-maze The zero-maze apparatus (56-cm diameter) is divided in four equal quadrants with two opposing open quadrants and two opposing closed quadrants with gray acrylic walls (13-cm height). At the beginning of the trial, the subject mouse was placed head facing the entrance of a closed arm and allowed to explore the ring for 5 min under high light intensity (200 Lux). The time spent in the open arms was scored a posteriori on video recordings by a trained experimenter.

\section{Cognition}

Novel object recognition test The novel object recognition test was performed in a rectangular arena $(20 \times 40$ $\mathrm{cm})$. The test consisted of three sessions under constant light intensity (15 Lux). During the habituation session, the mice explored freely the empty arena for $5 \mathrm{~min}$. For the training session, two identical objects were placed on each side of the arena and the mouse allowed to explore for $10 \mathrm{~min}$. For the test session, one of the objects was replaced by a novel object and the mouse allowed to explore the objects for $10 \mathrm{~min}$. The time spent exploring the familiar and novel objects by the subject animal were manually scored a posteriori on video recordings of the sessions. A recognition index was calculated as the percentage of the time spent exploring the novel object over the total time spent exploring both objects [79].

\section{Ex vivo patch-clamp electrophysiological recordings}

Electrophysiological recordings were performed on subgroups of control or $p$-Cresol-treated which had not been subjected to behavioral tests but nevertheless belonged to larger cohorts in which behavioral impairments in the $p$-Cresol group were observed. Mice were anesthetized (ketamine $(150 \mathrm{mg} / \mathrm{kg}) /$ xylazine $(10 \mathrm{mg} /$ $\mathrm{kg})$ ), trans-cardially perfused with artificial cerebrospinal fluid (aCSF solution: $\mathrm{NaCl} 119 \mathrm{mM}, \mathrm{KCl} 2.5 \mathrm{mM}$, $\mathrm{NaH}_{2} \mathrm{PO}_{4} 1.25 \mathrm{mM}, \mathrm{MgSO}_{4} 1.3 \mathrm{mM}, \mathrm{CaCl}_{2} 2.5 \mathrm{mM}$, $\mathrm{NaHCO}_{3} 26 \mathrm{mM}$, glucose $11 \mathrm{mM}$ ). Brains were removed 
from skull and sliced sagittally in $250-\mu \mathrm{m}$ sections using a HM650V vibratome (Microm, France). Sections encompassing VTA were placed in an ice-cold cutting solution ( $\mathrm{KCl} 2.5 \mathrm{mM}, \mathrm{NaH}_{2} \mathrm{PO}_{4} 1.25 \mathrm{mM}, \mathrm{MgSO}_{4} 10$ $\mathrm{mM}, \mathrm{CaCl}_{2} 0.5 \mathrm{mM}$, glucose $11 \mathrm{mM}$, sucrose $234 \mathrm{mM}$, $\mathrm{NaHCO}_{3} 26 \mathrm{mM}$ ) bubbled with $95 \% \mathrm{O}_{2} / 5 \% \mathrm{CO}_{2}$. Slices were then incubated in aCSF at $37^{\circ} \mathrm{C}$ for $1 \mathrm{~h}$, and then kept at RT. For recordings, sections were transferred in a thermo-controlled $\left(32-34{ }^{\circ} \mathrm{C}\right)$ recording chamber superfused with aCSF $(2.5 \mathrm{ml} / \mathrm{min})$. Spontaneous excitatory post-synaptic currents (sEPSCs) or excitability were measured using visualized whole-cell voltage-clamp and current-clamp recordings, respectively, using an upright microscope (Olympus France). Putative dopamine neurons were identified in the VTA using common criteria: anatomical localization, cell body size, broad action potential, and large Ih current [80], as previously described [81]. Current-clamp recordings were performed using a Multiclamp 700B device (Molecular Devices, Sunnyvale, CA). Signals were collected and stored using a Digidata 1440A converter and pCLAMP 10.2 software (Molecular Devices, CA, USA). In all cases, access resistance was monitored by a step at $-10 \mathrm{mV}(0.1 \mathrm{~Hz})$ and experiments were discarded if the access resistance increased more than 20\% (internal solution: K-D-gluconate 135 $\mathrm{mM}, \mathrm{NaCl} 5 \mathrm{mM}, \mathrm{MgCl}_{2} 2 \mathrm{mM}$, HEPES $10 \mathrm{mM}$, EGTA $0.5 \mathrm{mM}$, MgATP $2 \mathrm{mM}$, NaGTP 0.4 mM). Depolarizing $(0-300 \mathrm{pA})$ or hyperpolarizing $(0-450 \mathrm{pA}) 800 \mathrm{~ms}$ current steps were used to assess excitability and membrane properties of VTA dopamine neurons. SEPSCs were assessed in voltage-clamp mode at a voltage of $65 \mathrm{mV}$ in the presence of picrotoxin $(50 \mu \mathrm{M})$ using the same internal solution. Off-line analyses were performed using Clampfit 10.2 (Axon Instruments, USA).

\section{Fecal microbiota transplantation (FMT)}

The FMT protocol was adapted from [82]. For induction experiments $\left(\mathrm{FMT}^{p \text {-Cresol }}\right.$ vs. $\left.\mathrm{FMT}^{\text {Control}}\right)$, recipient C57BL/6J mice were received at 3 weeks of age and habituated for 5 days to our facility. From day 1 to 3, mice were gavaged with omeprazole (Esomeprazole, Biogaran, $50 \mathrm{mg} / \mathrm{Kg} / 24 \mathrm{~h}$ ) each morning to lower gastric acidity and favor survival of the inoculum. In the evening of day 3 , food was removed, but mice had ad libitum access to water. On day 4, mice received 5 consecutive gavages of $200 \mu \mathrm{L}$ of a laxative, reconstituted as recommended by the manufacturer (Moviprep, Norgine SAS, RueilMalmaison, France) at 1-h 30-min intervals. The FMT inoculum was prepared by pooling individual fecal pellets from $\mathrm{n}=15$ control or $\mathrm{n}=15$ mice treated with $p$ Cresol for 4 weeks (as described in the animal treatment section). Fecal pools were then weighted and homogenized in ice-cold $d_{d d} \mathrm{H}_{2} \mathrm{O}$ (weight:volume $=1: 50$ ). Fecal slurry was filtered over a $70-\mu \mathrm{m}$ mesh to remove fibers and clogs. Recipient mice received 3 consecutive gavages of $200 \mu \mathrm{L}$ of the filtered fecal slurry at $2 \mathrm{~h}$ intervals. After the last gavage, mice were returned to clean cages and had ad libitum access to food and water. Body weight was monitored on days 4,5 , and 7 to verify that the mice recovered from the FMT procedure. Three weeks post-FMT, mice were subjected to behavioral tests 2 days apart in the following order: dyadic social interactions, motor stereotypies, 3-chamber test, noveltysuppressed feeding test, and zero-maze.

For rescue experiments (FMT ${ }^{\text {Control}}$ ), mice were treated with $p$-Cresol for 4 weeks (as described in the animal treatment section), subjected to behavioral tests 2 days apart in the following order: direct social interactions, motor stereotypies, and 3-chamber test. $p$-Cresol treatment was stopped at the end of behavioral testing when control and $p$-Cresol-treated mice were subjected to the FMT procedure described above. The FMT inoculum was prepared by pooling individual fecal pellets from $\mathrm{n}=15$ control donor untreated male mice of the same age. Three weeks post-FMT, mice were subjected to behavioral tests 2 days apart in the following order: direct social interactions, motor stereotypies, and 3chamber test. The ex vivo electrophysiological recordings were also performed three weeks post-FMT on parallel sub-groups of mice which were not subjected to behavioral testing.

\section{Fecal microbiota composition analysis using 16S rRNA gene sequencing \\ Fecal DNA sample preparation}

Mice were individually placed for $10 \mathrm{~min}$ in a sterile empty plastic cage, and feces were collected in Eppendorf tubes in the morning (10-11 a.m.), snapped-frozen in liquid nitrogen, and stored at $-80{ }^{\circ} \mathrm{C}$ until further use. Genomic DNA was obtained from fecal samples using the QIAamp power fecal DNA kit (Qiagen), and DNA concentration was determined using a TECAN Fluorometer (Qubit ${ }^{\circ}$ dsDNA HS Assay Kit, Molecular Probes).

\section{S rRNA gene sequencing}

The V3-V4 hypervariable region of the $16 \mathrm{~S}$ rRNA gene was amplified by PCR using the following primers: a forward primer 5'-CTT TCC CTA CAC GAC GCT CTT CCG ATC TAC GGR AGG CAG CAG-3 (28-nt Illumina adapter (in bold) followed the $14 \mathrm{nt}$ broad range bacterial primer $343 \mathrm{~F}$ ) and a reverse primer $5^{\prime}$-GGA GTT CAG ACG TGT GCT CTT CCG ATC TTA CCA GGG TAT CTA ATC CT-3' (28-nt Illumina adapter (in bold) followed by the $19 \mathrm{nt}$ broad range bacterial primer 784R). The PCR reaction mix consisted of $10 \mathrm{ng}$ of fecal DNA template, $0.5 \mu \mathrm{M}$ primers, $0.2 \mathrm{mM} \mathrm{dNTP}$, and 0.5 $\mathrm{U}$ of the DNA-free Taq-polymerase (MolTaq 16S, 
Molzym, Bremen, Germany). The PCR cycles were as follow: 1 cycle at $94{ }^{\circ} \mathrm{C}$ for $60 \mathrm{~s}$, followed by 30 cycles at $94{ }^{\circ} \mathrm{C}$ for $60 \mathrm{~s}, 65^{\circ} \mathrm{C}$ for $60 \mathrm{~s}, 72{ }^{\circ} \mathrm{C}$ for $60 \mathrm{~s}$, and a final step at $72{ }^{\circ} \mathrm{C}$ for $10 \mathrm{~min}$. The PCR reactions were sent to the @Bridge platform (INRAe, Jouy-en-Josas, France) for sequencing (Illumina Miseq technology). Single multiplexing was performed using home-made $6 \mathrm{bp}$ index, which were added to R784 during a second PCR with 12 cycles using forward primer (AAT GAT ACG GCG ACC ACC GAG ATC TAC ACT CTT TCC CTA CAC GAC) and reverse primer (CAA GCA GAA GAC GGC ATA CGA GAT-index-GTG ACT GGA GTT CAG ACG TGT). The resulting PCR products were purified and loaded onto the Illumina MiSeq cartridge according to the manufacturer instructions. Run quality was checked internally using PhiX, and sequences assigned to the corresponding based on the $6 \mathrm{pb}$ index.

\section{Sequences preprocessing}

Demultiplexed reads were imported as a whole dataset into QIIME2 [83] (v. 2020.8) that was used for further processing [84]. Primer sequences were removed from reads with Cutadapt [85] and primer-deprived reads were discarded. Based on a sequencing quality score above 18 as recommended [86], forward and reverse reads were truncated at base position 236 and 225 respectively. Paired reads were then processed with QIIM E2 implementation of DADA2 [87]. Low-quality reads (with more than 2 expected errors) were dropped. Reads were then denoised by the pseudo-pooling method for finer low-count variant detection. Chimera reads were removed using the pooled option. Fifty-three percent of total reads passed quality control checks and were included in downstream analysis, yielding 2494 Amplicon Sequence Variants (ASV) of which 873 were detected at least in 2 individuals from each condition.

\section{Richness and diversity}

High-quality reads were aligned to QIIME2 reference library using mafft $[88,89]$. Aligned reads were masked in order to remove high variation reads. Then, a phylogenetic tree was constructed from the masked alignment of all ASV sequences with the QIIME2 implementation of FastTree [90]. Richness and evenness metrics were calculated with the QIIME2 "richness and diversity" plugin and observed richness', Shannon's and Pielou evenness' indexes were computed for each condition. Divergence in community composition between samples was quantitatively assessed through a compositional $\beta$-diversity metric rooted in a centered log ratio transformation and matrix completion called robust Aitchison PCA and implemented in QIIME2, which has superior performances to more classical abundance-based Bray-Curtis or Unifrac distances [91]. Aitchison's distances were calculated on non-rarefied compositional datasets using DEICODE based on 10,453 reads/sample, the largest sampling depth possible, that is robust with respect to compositional data with high levels of sparsity [91]. Hypothesis testing with the beta-group-significance was performed using PERMANOVA applied to the $\beta$-diversity ordination artifact using the native QIIME2 implementation of ADONIS from the vegan R package with 999 permutations. Sequences, ASV table, and rooted phylogenetic tree were extracted from QIIME2 for further analysis.

Taxonomic assignment To optimize taxonomic affiliation of the ASV, we used a three-step approach. First, taxonomy was inferred using Kraken2 algorithm that is more accurate and faster for 16S rRNA profiling data than the sklearn-classifier implemented in QIIME 2 [92]. Kraken 2 internal quality score was set at 0.05 as recommended [92]. To ascertain first-pass taxonomic affiliation, individual ASV sequences were aligned against the 16S rRNA sequences of their respective Kraken 2derived taxonomic affiliation using NCBI BLAST + from the non-recombinant nucleotide (nr/nt) database architecture [93]. Seventy-five percent of ASV yielded firstpass satisfactory taxonomic assignment with $>95 \%$ homology with their first-hit assigned sequence. The remaining $25 \%$ of ASV were blasted again against the next hit proposed by Kraken2 until a higher homology score was reached in a method inspired by [94]. After these iterative steps, we assigned up to $85 \%$ of all ASV with a homology score above $95 \%$. For the remaining unassigned ASV (15\%), their sequence was blasted on the $\mathrm{nr} / \mathrm{nt}$ database of bacterial sequences excluding uncultured and environmental sequences, yielding taxonomic assignation for $7 \%$ ASV more. At the end of the assignment process, $92 \%$ of ASV were taxonomically assigned with a homology score above 95\%. For the remaining $8 \%$ of ASV with no hit above $95 \%$ homology, the closest homology match sequence (including uncultured and environmental sequences) was recorded. Tab. S4 (Additional file 5) recapitulates the name and sequence of each of the ASVs detected in at least two individuals in each dataset, as well as the taxon identity and homology scores (percent of identity, bitscore, e-value) for the nearest homology match.

\section{Microbial composition analysis}

Individual datasets management (taxonomy, abundance, and metadata) was done on Phyloseq [95]. The effects of $p$-Cresol treatment or $\mathrm{FMT}^{p \text {-Cresol }}$ vs. $\mathrm{FMT}^{\text {Control }}$ were assessed using the analysis of composition of microbiomes (ANCOM) method [96]. ANCOM accounts for the compositional nature of $16 \mathrm{~S}$ rRNA gene sequencing datasets, is very sensitive for datasets $>20$ samples, and displays superior performances to control for false 
discovery rate compared to other methods such as DESeq2 [39]. The utility of ANCOM has been demonstrated for preprocessing sparse microbiome data sets with matrix completion to allow compositional ordination and to preserve information about the features driving differences among samples [39]. ANCOM accounts for compositionality using centered log ratio (CLR) analysis and therefore improves inference in microbiota survey data [39]. Recommended ANCOM thresholds were used to identify dysregulated taxa: $|C L R|>0.2$ and $\mathrm{W}=0.7$. Of note, the $p$-Cresol versus control dataset consisted of two batches of sequences obtained from two independent biological replicates $(\mathrm{n}=$ 15 animals/group/replicate) and the built-in batch effect correction implemented in ANCOM was used to account for batch effect. Based on features identified by ANCOM at each taxonomic level (from ASV to phylum), synthetic cladograms were built using GraPhlAn [97].

\section{Association between microbiota composition, behavioral scores, or fecal p-Cresol}

To assess relationships between changes in microbial distributions and behavioral variables or fecal $p$-Cresol), we used random forest regression models with nested cross-validation implemented in QIIME2. Independent models were built to identify ASV best predicting social interaction deficits, using the variable "total social contact time" or stereotypies using a composite variable obtained by summing the centered-scaled numbers of circling events and headshakes or fecal $p$-Cresol concentrations.

\section{Blast analysis of p-Cresol biosynthetic enzymes}

As references for queries, we used the protein sequence of ThiH (tyrosine lyase (NP 418417) from E. coli K-12 substrate MG1655), TyrB (tyrosine aminotransferase (NP 418478) from E. coli $\mathrm{K}-12$ substrate MG1655), and HpdA, HpdB, and HpdC ( $p$-hydroxyphenylacetate decarboxylase subunits A (AJ543427), B (AJ543425), and C (AJ543426) from C. difficile DSM 1296T) (Additional file 4: Tab. S3). Sequence homology search was performed using the Blast webtool set with default parameters on the $\mathrm{nr} / \mathrm{nt}$ database restricted to Duncaniella dubosii (taxid: 2518971) and the genera Barnesiella (taxid: 397864) and Anaerobium (taxid: 1855714). We considered as potential homologous enzymes sequences presenting amino acid identity above $30 \%$ along more than $50 \%$ of the query sequence.

\section{Statistics}

Comparisons between two groups were performed using 2-tailed unpaired Mann-Whitney's non-parametric Utest. Multiple group comparisons were performed using two-way ANOVA with factors stated in legends and text. Prior to ANOVA, normality of data was assessed using Kolmogorov-Smirnov's test. Post hoc comparisons were performed using Šidák's correction for multiple comparison. Detailed ANOVA statistics for each panel are provided (Additional file 6: Tab. S6). For comparison analysis of frequency distributions, KolmogorovSmirnov's test was used. Principal component analysis (PCA) of behavioral data was performed for each dataset consisting of scores from the dyadic social interaction test and scores from the direct monitoring of motor stereotypies using the webtool Clustvis [98]. Statistical significance was set according to a two-tailed p-value or adjusted (if relevant) $\mathrm{p}$-value $(\mathrm{p})<0.05$. Only significant differences are displayed on the graphs.

\section{Supplementary Information}

The online version contains supplementary material available at https://doi. org/10.1186/s40168-021-01103-z.

Additional file 1: Fig. S1. $p$-Cresol treatment does not impact general physiological parameters, increases urinary and fecal $p$-Cresol excretion but not its serum levels. Fig. S2. $p$-Cresol treatment-induced behavioral alterations: additional parameters in the 3-chamber, dyadic social interactions and stereotypies tests and results from tests assessing locomotor activity, anxiety or cognition (relative to Fig. 1). Fig. S3. Autistic-like behaviors persist after discontinuation of $p$-Cresol for 4 weeks. Fig. S4. Additional behavioral parameters for the 3-chamber, dyadic social interac tions, motor stereotypies tests and anxiety in FMTControl and FMTp-Cresol mice (relative to Fig. 4). Fig. S5. $p$-Cresol microbial biosynthetic pathways (relative to Fig. 5). Fig. S6. FMT experiments from control donors (FMTControl) to Control or $p$-Cresol-treated recipient mice: additional behavioral parameters in the 3-chamber, dyadic social interactions and stereotypies tests (relative to Fig. 6). Tab. S5: Recapitulation of the behavioral tests performed in the course of the study.

Additional file 2: Table S1. ANCOM analysis: significant microbial features (from ASV to phylum) discriminating $p$-Cresol from Control microbiota (relative to Fig. 3).

Additional file 3: Table S2. ANCOM analysis: significant microbial features (from ASV to phylum) discriminating FMTControl from FMTpCresol mice, 3 weeks post-FMT (relative to Fig. 5).

Additional file 4: Table S3. Output of blast sequence analysis for $\mathrm{ThiH}$, Tyr and $\mathrm{HpdA} / \mathrm{B} / \mathrm{C}$ enzymes involved in $\mathrm{p}$-Cresol synthesis (relative to Table 1).

Additional file 5: Table S4. Details on ASV sequences and taxonomic affiliation.

Additional file 6: Table S6. Compilation of ANOVA statistics for behavioral data analyses and PERMANOVA statistics for $16 \mathrm{~S}$ rRNA gene sequencing-based richness and diversity analyses.

\section{Acknowledgements}

We thank Lucien Relmy for expert technical assistance with animal care and Thomas Lorivel for help with behavioral tests set-up. We are grateful to Dominique Gauguier (Centre de Recherche des Cordeliers, Paris) for helpful discussions regarding the procedure for fecal microbiota transplantation.

\section{Authors' contributions}

PBM, JAJB, JCa, JCh, and JLM performed behavioral phenotyping and scoring. SPF, RCC, and JB performed electrophysiological recordings. BA, CC, and PL performed 165 rRNA sequencing. NC and SB performed the analysis of $16 \mathrm{~S}$ rRNA datasets. LMG, AM, and MED performed urinary and serum $p$ Cresol measures. JML and JCal performed fecal $p$-Cresol measures. LD designed the study, performed statistical analysis, and wrote the manuscript. 
NG contributed to study design and writing of the manuscript. The authors read and approved the final manuscript.

\section{Funding}

LD and MED are grateful to the European Community $7^{\text {th }}$ Framework Program under Coordinated Action NEURON-ERANET (grant agreement 291840). ANR and MRC (MR/M501797/1). LD thanks the ANR (ANR-19-CE140024-MicrobiAutism). JAJB and JLM thank Region the Centre-Val de Loire (ARD2020 Biomedicaments GPCRAb) and the Labex Mablmprove for financial support. Funding agencies were not involved in the design of the study and collection, analysis, and interpretation of data and in writing the manuscript. JC is funded by a PhD fellowship from the Fondation de la Recherche Médicale (FRM reference: ECO202006011591).

\section{Availability of data and materials}

The $16 \mathrm{~S}$ rRNA gene sequencing datasets supporting the conclusions of this article are available in the NCBI repository (BioProject \# PRJNA724745). Hyperlink to datasets:

https://dataview.ncbi.nlm.nih.gov/object/PRJNA724745?reviewer=rg8fg4 7p0fh662g55oi1qnp3eo

\section{Declarations}

\section{Ethics approval and consent to participate}

Animal housing and experimentation were conducted in facilities certified by regional authorities (Direction Départementale de Protection des Populations des Alpes-Maritimes, accreditation \#EC-06-152-5). The procedures involved in this study were approved by the local ethics committee for animal experimentation (Ciepal-Azur) and the Ministère de l'Enseignement Supérieur et de la Recherche, in agreement with the European Communities Council Directive (2010/63EU) for animal experiments.

\section{Consent for publication}

Not applicable.

\section{Competing interests}

The authors declare that they have no competing interests.

\section{Author details}

${ }^{1}$ Institut de Pharmacologie Moléculaire et Cellulaire, Centre National de la Recherche Scientifique, Université Côte d'Azur, 660 route des Lucioles, 06560 Valbonne, France. ${ }^{2}$ Physiologie de la Reproduction et des Comportements, UMR0075 INRAE, UMR7247 CNRS, IFCE, Inserm, Université François Rabelais, 37380 Nouzilly, France. ${ }^{3}$ UMR 1253, iBrain, Université de Tours, Inserm, CNRS, Tours 37200, France. ${ }^{4}$ Current address: Structural Biology, Radiation Facility, European Synchrotron, Grenoble, France. ${ }^{5}$ Division of Systems Medicine, Department of Metabolism, Digestion and Reproduction, Faculty of Medicine, Imperial College London, London SW7 2AZ, UK. ${ }^{6}$ Genomic and Environmental Medicine, National Heart \& Lung Institute, Faculty of Medicine, Imperial College London, London SW3 6KY, UK. ${ }^{7}$ European Genomic Institute for Diabetes, CNRS UMR 8199, INSERM UMR 1283, Institut Pasteur de Lille, Lille University Hospital, University of Lille, 59045 Lille, France. ${ }^{8}$ McGill University and Genome Quebec Innovation Centre, 740 Doctor Penfield Avenue, Montréal, QC H3A 0G1, Canada. ${ }^{9}$ AgroParisTech, INRAE, Institut Micalis, Université Paris-Saclay, Jouy-en-Josas, France. ${ }^{10}$ UMR-S 942, INSERM, Department of Biochemistry, Lariboisière Hospital, Paris, France. ${ }^{11}$ Centre for Biological Resources, BB-0033-00064, Lariboisière Hospital, Paris, France. ${ }^{12}$ Fondation FondaMental, Créteil, France.

Received: 29 January 2021 Accepted: 27 May 2021

Published online: 08 July 2021

\section{References}

1. Vuong $\mathrm{HE}$, Hsiao EY. Emerging roles for the gut microbiome in autism spectrum disorder. Biol Psychiatry. 2017:81(5):411-23. https://doi.org/10.101 6/j.biopsych.2016.08.024.

2. Fombonne E, MacFarlane $H$, Salem AC. Epidemiological surveys of ASD: advances and remaining challenges. J Autism Dev Disord. 2021. https://doi. org/10.1007/s10803-021-05005-9.
3. Bourgeron T. Current knowledge on the genetics of autism and propositions for future research. C R Biol. 2016;339(7-8):300-7. https://doi. org/10.1016/j.crvi.2016.05.004.

4. Lai MC, Lombardo MV, Baron-Cohen S. Autism. Lancet. 2014;383(9920):896910. https://doi.org/10.1016/S0140-6736(13)61539-1.

5. Mazurek MO, Vasa RA, Kalb LG, Kanne SM, Rosenberg D, Keefer A, et al. Anxiety, sensory over-responsivity, and gastrointestinal problems in children with autism spectrum disorders. J Abnorm Child Psychol. 2013;41(1):165-76. https://doi.org/10.1007/s10802-012-9668-x.

6. Nikolov RN, Bearss KE, Lettinga J, Erickson C, Rodowski M, Aman MG, et al. Gastrointestinal symptoms in a sample of children with pervasive developmental disorders. J Autism Dev Disord. 2009;39(3):405-13. https:// doi.org/10.1007/s10803-008-0637-8.

7. Gorrindo P, Williams KC, Lee EB, Walker LS, McGrew SG, Levitt P. Gastrointestinal dysfunction in autism: parental report, clinical evaluation, and associated factors. Autism Res. 2012;5(2):101-8. https://doi.org/10.1 002/aur.237.

8. Xu M, Xu X, Li J, Li F. Association between gut microbiota and autism spectrum disorder: a systematic review and meta-analysis. Front Psychiatry. 2019;10:473. https://doi.org/10.3389/fpsyt.2019.00473.

9. Iglesias-Vazquez L, Van Ginkel Riba G, Arija V, Canals J: Composition of gut microbiota in children with autism spectrum disorder: a systematic review and meta-analysis. Nutrients. 2020;12(3):792. https://doi.org/10.3390/nu1203 0792.

10. Liu F, Li J, Wu F, Zheng H, Peng Q, Zhou H. Altered composition and function of intestinal microbiota in autism spectrum disorders: a systematic review. Transl Psychiatry. 2019;9(1):43. https://doi.org/10.1038/s41398-019-03 89-6.

11. Kang DW, Adams JB, Coleman DM, Pollard EL, Maldonado J, MCDonoughMeans $\mathrm{S}$, et al. Long-term benefit of microbiota transfer therapy on autism symptoms and gut microbiota. Sci Rep. 2019;9(1):5821. https://doi.org/10.1 038/s41598-019-42183-0.

12. Emond P, Mavel S, Aidoud N, Nadal-Desbarats L, Montigny F, BonnetBrilhault F, et al. GC-MS-based urine metabolic profiling of autism spectrum disorders. Anal Bioanal Chem. 2013;405(15):5291-300. https://doi.org/10.1 007/s00216-013-6934-x.

13. Yap IK, Angley M, Veselkov KA, Holmes E, Lindon JC, Nicholson JK. Urinary metabolic phenotyping differentiates children with autism from their unaffected siblings and age-matched controls. J Proteome Res. 2010;9(6): 2996-3004. https://doi.org/10.1021/pr901188e.

14. Altieri L, Neri C, Sacco R, Curatolo P, Benvenuto A, Muratori F, et al. Urinary pcresol is elevated in small children with severe autism spectrum disorder. Biomarkers. 2011;16(3):252-60. https://doi.org/10.3109/1354750X.2010.548010.

15. Gabriele S, Sacco R, Cerullo S, Neri C, Urbani A, Tripi G, et al. Urinary p-cresol is elevated in young French children with autism spectrum disorder: a replication study. Biomarkers. 2014;19(6):463-70. https://doi.org/10.3109/13 54750X.2014.936911

16. Dieme B, Mavel S, Blasco H, Tripi G, Bonnet-Brilhault F, Malvy J, et al. Metabolomics study of urine in autism spectrum disorders using a multiplatform analytical methodology. J Proteome Res. 2015;14(12):5273-82. https://doi.org/10.1021/acs.jproteome.5b00699.

17. Macfabe DF. Short-chain fatty acid fermentation products of the gut microbiome: implications in autism spectrum disorders. Microbial ecology in health and disease. 2012;23(0). https://doi.org/10.3402/mehd.v23i0.19260.

18. De Angelis M, Piccolo M, Vannini L, Siragusa S, De Giacomo A, Serrazzanetti $\mathrm{Dl}$, et al. Fecal microbiota and metabolome of children with autism and pervasive developmental disorder not otherwise specified. Plos One. 2013; 8(10):e76993. https://doi.org/10.1371/journal.pone.0076993.

19. Kang DW, Ilhan ZE, Isern NG, Hoyt DW, Howsmon DP, Shaffer M, et al. Differences in fecal microbial metabolites and microbiota of children with autism spectrum disorders. Anaerobe. 2017:49:121-31.

20. Hsiao EY, McBride SW, Hsien S, Sharon G, Hyde ER, McCue T, et al. Microbiota modulate behavioral and physiological abnormalities associated with neurodevelopmental disorders. Cell. 2013;155(7):1451-63. https://doi. org/10.1016/j.cell.2013.11.024.

21. Buffington SA, Di Prisco GV, Auchtung TA, Ajami NJ, Petrosino JF, CostaMattioli M. Microbial reconstitution reverses maternal diet-induced social and synaptic deficits in offspring. Cell. 2016;165(7):1762-75. https://doi.org/1 0.1016/j.cell.2016.06.001.

22. Sgritta M, Dooling SW, Buffington SA, Momin EN, Francis MB, Britton RA, et al. Mechanisms underlying microbial-mediated changes in social 
behavior in mouse models of autism spectrum disorder. Neuron. 2019; 101(2):246-59 e246. https://doi.org/10.1016/j.neuron.2018.11.018.

23. Golubeva AV, Joyce SA, Moloney G, Burokas A, Sherwin E, Arboleya S, et al. Microbiota-related changes in bile acid \& tryptophan metabolism are associated with gastrointestinal dysfunction in a mouse model of autism. EBioMedicine. 2017;24:166-78. https://doi.org/10.1016/j.ebiom.2017.09.020.

24. de Theije CG, Wopereis H, Ramadan M, van Eijndthoven T, Lambert J, Knol J, et al. Altered gut microbiota and activity in a murine model of autism spectrum disorders. Brain Behav Immun. 2014;37:197-206. https://doi.org/1 0.1016/j.bbi.2013.12.005.

25. Sharon G, Cruz NJ, Kang DW, Gandal MJ, Wang B, Kim YM, et al. Human gut microbiota from autism spectrum disorder promote behavioral symptoms in mice. Cell. 2019;177(6):1600-18 e1617. https://doi.org/10.1016/j.cell.2019. 05.004 .

26. Needham BD, Kaddurah-Daouk R, Mazmanian SK: Gut microbial molecules in behavioural and neurodegenerative conditions. Nat Rev Neurosci. 2020; 21(12):717-31. https://doi.org/10.1038/s41583-020-00381-0. Epub 2020 Oct 16.

27. Needham BD, Adame MD, Serena G, Rose DR, Preston GM, Conrad MC, et al. Plasma and fecal metabolite profiles in autism spectrum disorder. Biol Psychiatry. 2021;89(5):451-62. https://doi.org/10.1016/j.biopsych.2020.09.025.

28. Gevi F, Belardo A, Zolla L. A metabolomics approach to investigate urine levels of neurotransmitters and related metabolites in autistic children. Biochim Biophys Acta Mol Basis Dis. 1866;2020(10):165859.

29. Persico AM, Napolioni V. Urinary p-cresol in autism spectrum disorder. Neurotoxicol Teratol. 2013;36:82-90. https://doi.org/10.1016/j.ntt.2012.09.002.

30. Saito Y, Sato T, Nomoto K, Tsuji H: Identification of phenol- and p-cresolproducing intestinal bacteria by using media supplemented with tyrosine and its metabolites. FEMS Microbiol Ecol. 2018;94(9):fiy125. https://doi.org/1 0.1093/femsec/fiy 125 .

31. Passmore IJ, Letertre MPM, Preston MD, Bianconi I, Harrison MA, Nasher F, et al. Para-cresol production by Clostridium difficile affects microbial diversity and membrane integrity of Gram-negative bacteria. Plos Pathog. 2018;14(9): e1007191. https://doi.org/10.1371/journal.ppat.1007191.

32. Hung LW, Neuner S, Polepalli JS, Beier KT, Wright M, Walsh JJ, et al. Gating of social reward by oxytocin in the ventral tegmental area. Science. 2017; 357(6358):1406-11. https://doi.org/10.1126/science.aan4994.

33. Supekar K, Kochalka J, Schaer M, Wakeman H, Qin S, Padmanabhan A, et al. Deficits in mesolimbic reward pathway underlie social interaction impairments in children with autism. Brain. 2018;141(9):2795-805. https:// doi.org/10.1093/brain/awy191.

34. Krishnan V, Stoppel DC, Nong Y, Johnson MA, Nadler MJ, Ozkaynak E, et al. Autism gene Ube3a and seizures impair sociability by repressing VTA CbIn 1. Nature. 2017:543(7646):507-12. https://doi.org/10.1038/nature21678.

35. Bariselli S, Hornberg H, Prevost-Solie C, Musardo S, Hatstatt-Burkle L, Scheiffele $P$, et al. Role of VTA dopamine neurons and neuroligin 3 in sociability traits related to nonfamiliar conspecific interaction. Nat Commun. 2018;9(1):3173. https://doi.org/10.1038/s41467-018-05382-3.

36. Bariselli S, Tzanoulinou S, Glangetas C, Prevost-Solie C, Pucci L, Viguie J, et al. SHANK3 controls maturation of social reward circuits in the VTA. Nat Neurosci. 2016;19(7):926-34. https://doi.org/10.1038/nn.4319.

37. Lecca S, Luchicchi A, Scherma M, Fadda P, Muntoni AL, Pistis M. Delta(9)tetrahydrocannabinol during adolescence attenuates disruption of dopamine function induced in rats by maternal immune activation. Front Behav Neurosci. 2019;13:202. https://doi.org/10.3389/fnbeh.2019.00202.

38. Mandal S, Van Treuren W, White RA, Eggesbo M, Knight R, Peddada SD. Analysis of composition of microbiomes: a novel method for studying microbial composition. Microb Ecol Health Dis. 2015;26:27663.

39. Weiss S, Xu ZZ, Peddada S, Amir A, Bittinger K, Gonzalez A, et al. Normalization and microbial differential abundance strategies depend upon data characteristics. Microbiome. 2017;5(1):27. https://doi.org/10.1186/s401 68-017-0237-y.

40. Challand MR, Martins FT, Roach PL. Catalytic activity of the anaerobic tyrosine lyase required for thiamine biosynthesis in Escherichia coli. J Biol Chem. 2010;285(8):5240-8. https://doi.org/10.1074/jbc.M109.056606.

41. Jaglin M, Rhimi M, Philippe C, Pons N, Bruneau A, Goustard B, et al. Indole, a signaling molecule produced by the gut microbiota, negatively impacts emotional behaviors in rats. Front Neurosci. 2018;12:216. https://doi.org/1 0.3389/fnins.2018.00216.

42. Mir HD, Milman A, Monnoye M, Douard V, Philippe C, Aubert A, et al. The gut microbiota metabolite indole increases emotional responses and adrenal medulla activity in chronically stressed male mice. Psychoneuroendocrinology. 2020;119:104750. https://doi.org/10.1016/j. psyneuen.2020.104750.

43. Pascucci T, Colamartino M, Fiori E, Sacco R, Coviello A, Ventura R, PuglisiAllegra S, Turriziani L, Persico AM: P-cresol alters brain dopamine metabolism and exacerbates autism-like behaviors in the BTBR mouse. Brain Sci. 2020;10(4):233. https://doi.org/10.3390/brainsci10040233.

44. Lagkouvardos I, Pukall R, Abt B, Foesel BU, Meier-Kolthoff JP, Kumar N, et al. The Mouse Intestinal Bacterial Collection (miBC) provides host-specific insight into cultured diversity and functional potential of the gut microbiota. Nat Microbiol. 2016;1(10):16131. https://doi.org/10.1038/ nmicrobiol.2016.131

45. Xiao L, Feng Q, Liang S, Sonne SB, Xia Z, Qiu X, et al. A catalog of the mouse gut metagenome. Nature biotechnology. 2015;33(10):1103-8. https://doi.org/10.1038/nbt.3353.

46. Pellissier LP, Gandia J, Laboute T, Becker JAJ, Le Merrer J. mu opioid receptor, social behaviour and autism spectrum disorder: reward matters. $\mathrm{Br}$ J Pharmacol. 2018;175(14):2750-69. https://doi.org/10.1111/bph.13808.

47. Ranaldi R. Dopamine and reward seeking: the role of ventral tegmental area. Rev Neurosci. 2014;25(5):621-30. https://doi.org/10.1515/revneuro-2 014-0019.

48. Gunaydin LA, Grosenick L, Finkelstein JC, Kauvar IV, Fenno LE, Adhikari A, et al. Natural neural projection dynamics underlying social behavior. Cell. 2014;157(7):1535-51. https://doi.org/10.1016/j.cell.2014.05.017.

49. Kim H, Lim CS, Kaang BK. Neuronal mechanisms and circuits underlying repetitive behaviors in mouse models of autism spectrum disorder. Behav Brain Funct. 2016;12(1):3. https://doi.org/10.1186/s12993-016-0087-y.

50. Lewis MH, Tanimura Y, Lee LW, Bodfish JW. Animal models of restricted repetitive behavior in autism. Behav Brain Res. 2007;176(1):66-74. https://doi. org/10.1016/j.bbr.2006.08.023.

51. Kim CH. Immune regulation by microbiome metabolites. Immunology. 2018;154(2):220-9. https://doi.org/10.1111/imm.12930.

52. Venkatesh M, Mukherjee S, Wang H, Li H, Sun K, Benechet AP, et al. Symbiotic bacterial metabolites regulate gastrointestinal barrier function via the xenobiotic sensor PXR and Toll-like receptor 4. Immunity. 2014;41(2): 296-310. https://doi.org/10.1016/j.immuni.2014.06.014.

53. Bolognini D, Tobin AB, Milligan G, Moss CE. The pharmacology and function of receptors for short-chain fatty acids. Mol Pharmacol. 2016;89(3):388-98. https://doi.org/10.1124/mol.115.102301.

54. Swann JR, Spitzer SO, Diaz Heijtz R: Developmental signatures of microbiota-derived metabolites in the mouse brain. Metabolites. 2020;10(5): 172. https://doi.org/10.3390/metabo10050172.

55. Vuong HE, Pronovost GN, Williams DW, Coley EJL, Siegler EL, Qiu A, et al. The maternal microbiome modulates fetal neurodevelopment in mice. Nature. 2020;586(7828):281-6. https://doi.org/10.1038/s41586-020-2745-3.

56. Vanholder R, Bammens B, de Loor H, Glorieux G, Meijers B, Schepers E, et al. Warning: the unfortunate end of p-cresol as a uraemic toxin. Nephrol Dial Transplant. 2011;26(5):1464-7. https://doi.org/10.1093/ndt/gfr056.

57. Gryp T, Vanholder R, Vaneechoutte M, Glorieux G: p-Cresyl sulfate. Kidney Int. 2020;97(6):1230-42. https://doi.org/10.1016/j.kint.2020.01.028. Epub 2020 Feb 17.

58. Fulling C, Dinan TG, Cryan JF. Gut microbe to brain signaling: what happens in vagus. Neuron. 2019;101(6):998-1002. https://doi.org/10.1016/j.neuron.201 9.02.008.

59. Gonzalez-Arancibia C, Urrutia-Pinones J, Illanes-Gonzalez J, Martinez-Pinto J, Sotomayor-Zarate R, Julio-Pieper M, et al. Do your gut microbes affect your brain dopamine? Psychopharmacology (Berl). 2019;236(5):1611-22. https:// doi.org/10.1007/s00213-019-05265-5.

60. Han W, Tellez LA, Perkins MH, Perez IO, Qu T, Ferreira J, et al. A neural circuit for gut-induced reward. Cell. 2018;175(3):887-8. https://doi.org/10.1016/j. cell.2018.10.018.

61. Loomes R, Hull L, Mandy WPL. What is the male-to-female ratio in autism spectrum disorder? A systematic review and meta-analysis. J Am Acad Child Adolesc Psychiatry. 2017;56(6):466-74. https://doi.org/10.1016/j.jaac.2017.03.013.

62. Fiehn O. Metabolomics by gas chromatography-mass spectrometry: combined rargeted and untargeted profiling. Curr Protoc Mol Biol. 2016;114: $303431-2$

63. Gao X, Pujos-Guillot E, Martin JF, Galan P, Juste C, Jia W, et al. Metabolite analysis of human fecal water by gas chromatography/mass spectrometry with ethyl chloroformate derivatization. Anal Biochem. 2009;393(2):163-75 https://doi.org/10.1016/j.ab.2009.06.036. 
64. Korytowska N, Wyczalkowska-Tomasik A, Wisniewska A, Paczek L, Giebultowicz J. Development of the LC-MS/MS method for determining the p-cresol level in plasma. J Pharm Biomed Anal. 2019;167:149-54. https://doi. org/10.1016/j.jpba.2019.01.041.

65. Santa T. Derivatization reagents in liquid chromatography/electrospray ionization tandem mass spectrometry. Biomed Chromatogr. 2011;25(1-2):110. https://doi.org/10.1002/bmc.1548.

66. Becker JA, Clesse D, Spiegelhalter C, Schwab Y, Le Merrer J, Kieffer BL. Autistic-like syndrome in mu opioid receptor null mice is relieved by facilitated mGluR4 activity. Neuropsychopharmacology. 2014;39(9):2049-60. https://doi.org/10.1038/npp.2014.59.

67. Pujol CN, Pellissier LP, Clement C, Becker JAJ, Le Merrer J. Back-translating behavioral intervention for autism spectrum disorders to mice with blunted reward restores social abilities. Transl Psychiatry. 2018;8(1):197. https://doi. org/10.1038/s41398-018-0247-y.

68. Leboucher A, Bermudez-Martin P, Mouska X, Amri EZ, Pisani DF, Davidovic L. Fmr1-deficiency impacts body composition, skeleton, and bone microstructure in a mouse model of fragile $X$ syndrome. Front Endocrinol (Lausanne). 2019;10:678. https://doi.org/10.3389/fendo.2019.00678.

69. Silverman JL, Yang M, Lord C, Crawley JN. Behavioural phenotyping assays for mouse models of autism. Nature reviews Neuroscience. 2010;11(7):490502. https://doi.org/10.1038/nrn2851.

70. Becker JAJ, Kieffer BL, Le Merrer J. Differential behavioral and molecular alterations upon protracted abstinence from cocaine versus morphine, nicotine. THC and alcohol. Addict Biol. 2017;22(5):1205-17. https://doi.org/1 $0.1111 / a d b .12405$.

71. Matsuo N, Tanda K, Nakanishi K, Yamasaki N, Toyama K, Takao K, et al. Comprehensive behavioral phenotyping of ryanodine receptor type 3 (RyR3) knockout mice: decreased social contact duration in two social interaction tests. Front Behav Neurosci. 2009:3:3.

72. Katayama Y, Nishiyama M, Shoji H, Ohkawa Y, Kawamura A, Sato T, et al. CHD8 haploinsufficiency results in autistic-like phenotypes in mice. Nature. 2016:537(7622):675-9. https://doi.org/10.1038/nature19357.

73. Spencer CM, Alekseyenko O, Serysheva E, Yuva-Paylor LA, Paylor R. Altered anxiety-related and social behaviors in the Fmr1 knockout mouse model of fragile X syndrome. Genes Brain Behav. 2005:4(7):420-30. https://doi.org/1 0.1111/j.1601-183X.2005.00123.x.

74. Thomas A, Burant A, Bui N, Graham D, Yuva-Paylor LA, Paylor R. Marble burying reflects a repetitive and perseverative behavior more than noveltyinduced anxiety. Psychopharmacology (Berl). 2009;204(2):361-73. https://doi. org/10.1007/s00213-009-1466-y.

75. Moustgaard A, Hau J, Lind NM. Effects of dopamine D4 receptor antagonist on spontaneous alternation in rats. Behav Brain Funct. 2008;4(1):49. https:// doi.org/10.1186/1744-9081-4-49.

76. Le Marec N, Ethier K, Rompre PP, Godbout R. Involvement of the medial prefrontal cortex in two alternation tasks using different environments. Brain Cogn. 2002:48(2-3):432-6.

77. Delotterie D, Ruiz G, Brocard J, Schweitzer A, Roucard C, Roche Y, et al. Chronic administration of atypical antipsychotics improves behavioral and synaptic defects of STOP null mice. Psychopharmacology (Berl). 2010;208(1): 131-41. https://doi.org/10.1007/s00213-009-1712-3.

78. Meirsman AC, Le Merrer J, Pellissier LP, Diaz J, Clesse D, Kieffer BL, et al. Mice lacking GPR88 show motor deficit, improved spatial learning, and low anxiety reversed by delta opioid antagonist. Biol Psychiatry. 2016;79(11):91727. https://doi.org/10.1016/j.biopsych.2015.05.020.

79. Fernandez SP, Muzerelle A, Scotto-Lomassese S, Barik J, Gruart A, DelgadoGarcia JM, et al. Constitutive and acquired serotonin deficiency alters memory and hippocampal synaptic plasticity. Neuropsychopharmacology. 2017;42(2):512-23. https://doi.org/10.1038/npp.2016.134.

80. Ungless MA, Grace AA. Are you or aren't you? Challenges associated with physiologically identifying dopamine neurons. Trends Neurosci. 2012;35(7): 422-30. https://doi.org/10.1016/j.tins.2012.02.003.

81. Fernandez SP, Broussot L, Marti F, Contesse T, Mouska X, Soiza-Reilly M, et al. Mesopontine cholinergic inputs to midbrain dopamine neurons drive stress-induced depressive-like behaviors. Nat Commun. 2018;9(1):4449. https://doi.org/10.1038/s41467-018-06809-7.

82. Le Roy T, Debedat J, Marquet F, Da-Cunha C, Ichou F, Guerre-Millo M, et al. Comparative evaluation of microbiota engraftment following fecal microbiota transfer in mice models: age, kinetic and microbial status matter. Front Microbiol. 2018;9:3289.
83. Bolyen E, Rideout JR, Dillon MR, Bokulich NA, Abnet CC, Al-Ghalith GA, et al. Reproducible, interactive, scalable and extensible microbiome data science using QIIME 2. Nature biotechnology. 2019;37(8):852-7. https://doi.org/10.1 038/s41587-019-0209-9.

84. Estaki M, Jiang L, Bokulich NA, McDonald D, Gonzalez A, Kosciolek T, et al. QIIME 2 enables comprehensive end-to-end analysis of diverse microbiome data and comparative studies with publicly available data. Curr Protoc Bioinformatics. 2020;70(1):e100. https://doi.org/10.1002/cpbi.100.

85. Kechin A, Boyarskikh U, Kel A. Filipenko M: cutPrimers: a new tool for accurate cutting of primers from reads of targeted next generation sequencing. J Comput Biol. 2017;24(11):1138-43. https://doi.org/10.1089/ cmb.2017.0096.

86. Mohsen A, Park J, Chen YA, Kawashima H, Mizuguchi K. Impact of quality trimming on the efficiency of reads joining and diversity analysis of Illumina paired-end reads in the context of QIIME1 and QIIME2 microbiome analysis frameworks. BMC Bioinformatics. 2019;20(1):581. https://doi.org/10.1186/s12 859-019-3187-5.

87. Callahan BJ, McMurdie PJ, Rosen MJ, Han AW, Johnson AJ, Holmes SP. DADA2: high-resolution sample inference from Illumina amplicon data. Nat Methods. 2016;13(7):581-3. https://doi.org/10.1038/nmeth.3869.

88. Kuraku S, Zmasek CM, Nishimura O, Katoh K. aLeaves facilitates on-demand exploration of metazoan gene family trees on MAFFT sequence alignment server with enhanced interactivity. Nucleic Acids Res. 2013;41(Web Server issue):W22-8.

89. Katoh K, Rozewicki J, Yamada KD. MAFFT online service: multiple sequence alignment, interactive sequence choice and visualization. Brief Bioinform. 2019;20(4):1160-6. https://doi.org/10.1093/bib/bbx108.

90. Price MN, Dehal PS, Arkin AP. FastTree: computing large minimum evolution trees with profiles instead of a distance matrix. Mol Biol Evol. 2009;26(7): 1641-50. https://doi.org/10.1093/molbev/msp077.

91. Martino C, Morton JT, Marotz CA, Thompson LR, Tripathi A, Knight R, Zengler K: A novel sparse compositional technique reveals microbial perturbations. mSystems. 2019;4(1):e00016-19. https://doi.org/10.1128/ mSystems.00016-19. eCollection Jan-Feb 2019.

92. Lu J, Salzberg SL. Ultrafast and accurate $16 \mathrm{~S}$ rRNA microbial community analysis using Kraken 2. Microbiome. 2020;8(1):124. https://doi.org/10.1186/ s40168-020-00900-2.

93. Camacho C, Coulouris G, Avagyan V, Ma N, Papadopoulos J, Bealer K, et al. BLAST+: architecture and applications. BMC Bioinformatics. 2009;10(1):421. https://doi.org/10.1186/1471-2105-10-421.

94. Bazinet AL, Ondov BD, Sommer DD, Ratnayake S. BLAST-based validation of metagenomic sequence assignments. PeerJ. 2018;6:e4892. https://doi.org/1 $0.7717 /$ peerj.4892.

95. McMurdie PJ, Holmes S. phyloseq: an R package for reproducible interactive analysis and graphics of microbiome census data. Plos One. 2013;8(4): e61217. https://doi.org/10.1371/journal.pone.0061217.

96. Kaul A, Mandal S, Davidov O, Peddada SD. Analysis of microbiome data in the presence of excess zeros. Front Microbiol. 2017:8:2114. https://doi.org/1 0.3389/fmicb.2017.02114

97. Asnicar F, Weingart G, Tickle TL, Huttenhower C, Segata N. Compact graphical representation of phylogenetic data and metadata with GraPhIAn. PeerJ. 2015;3:e1029. https://doi.org/10.7717/peerj.1029.

98. Metsalu T, Vilo J. ClustVis: a web tool for visualizing clustering of multivariate data using principal component analysis and heatmap. Nucleic Acids Res. 2015;43(W1):W566-70. https://doi.org/10.1093/nar/gkv468.

\section{Publisher's Note}

Springer Nature remains neutral with regard to jurisdictional claims in published maps and institutional affiliations. 